\title{
Emission and absorption lines of gamma-ray bursts affected by the relativistic motion of fireball ejecta ${ }^{\star}$
}

\author{
Yi-Ping Qin ${ }^{\star \star}$ \\ National Astronomical Observatories/Yunnan Observatory, Chinese Academy of Sciences, PO Box 110, Kunming, \\ Yunnan 650011, PR China
}

Received 29 November 2002 / Accepted 12 May 2003

\begin{abstract}
We display by numerical calculation how rest frame spectral lines appear in the observed spectrum of gamma-ray bursts due to the Doppler effect in the fireball framework. The analysis shows that: a) in the spectrum of a relativistically expanding fireball, all rest frame lines would shift to higher energy bands and would be significantly smoothed; b) rest frame weak narrow emission lines as well as narrow absorption lines and absorption line forests would be smoothed and would hardly be detectable; c) the features of rest frame broad emission lines as well as both strong and weak broad absorption lines would remain almost unchanged and therefore would be easier to detect; d) deep gaps caused by rest frame broad absorption lines would be significantly filled; e) a rest frame emission line forest would form a single broad line feature; f) the observed relative width of the rest frame very narrow line would approach $0.162 ; \mathrm{g}$ ) when the Lorentz factor $\Gamma$ is large enough, the observed line frequency $v_{\text {line }}$ and the rest frame line frequency $v_{0 \text {,line }}$ would be related by $v_{\text {line }} \approx 2 \Gamma v_{0, \text { line }}$. We also investigate the effect of time dependence of the line intensity and the effect of variation of $\Gamma$. We find that the feature of rest frame dimming narrow emission lines would disappear when $\Gamma$ is very large. The form of emission lines would be sharp on both edges when $\Gamma$ varies with time. This phenomenon depends not only on the initial Lorentz factor but also on the observation time.
\end{abstract}

Key words. gamma-rays: bursts - gamma-rays: theory - radiation mechanisms: nonthermal - relativity

\section{Introduction}

Due to the observed great output rate of radiation of gamma-ray bursts (GRBs), most models of the objects envision an expanding fireball (see e.g., Goodman 1986; Paczynski 1986). The gamma-ray emission would arise after the fireball becomes optically thin, in shocks produced when the ejecta collide with an external medium or in shocks occurred within a relativistic internal wind (Rees \& Meszaros 1992, 1994; Meszaros \& Rees 1993, 1994; Katz 1994; Paczynski \& Xu 1994; Sari et al. 1996). As the expanding motion of the outer shell of the fireball would be relativistic, the Doppler effect must be at work. As pointed out by Krolik \& Pier (1991), relativistic bulk motion of the gamma-ray-emitting plasma can account for some phenomena of bursts. However, in many cases, the whole fireball surface should be considered, and accounting for the effect the fireball surface itself would play a role (Meszaros \& Rees 1998; Qin 2002, hereafter Paper I).

As revealed by the observation of absorption lines of afterglows (Metzger et al. 1997), GRBs are confirmed to be events occurring within the environment of stars. Based on the assumption that there might be a strong magnitude field within the fireball and that the expansion would be relativistic, it was

* Appendices A, B and C are only available in electronic form at http://www . edpsciences.org

$\star \star$ e-mail: ypqin@public.km.yn.cn believed that synchrotron radiation would become a dominate mechanism (Liang et al. 1983). Unfortunately, the spectra observed so far are so different that none of the mechanisms proposed can account for most of the spectral data of the objects (Band et al. 1993; Schaefer et al. 1994; Preece et al. 1998, 2000).

Based on the assumption of the environment of stars, mechanisms of formation of emission as well as absorption lines for GRBs have been well established. Meszaros \& Rees (1998) suggested that gamma-ray burst outflows may entrain small blobs or filaments of dense, highly ionized metal-rich material, which might be accelerated by the flow to Lorentz factors in the range 10-100. In the event of neutron-star collisions or black hole-neutron star collisions, neutrino-antineutrino annihilation can produce electron-positron pairs. Thus it would be natural if there exist in the outer shell of fireballs some high energy emission lines such as the $6.4 \mathrm{keV}$ line and the $511 \mathrm{keV}$ annihilation line (Ramaty \& Meszaros 1981). As reviewed by Piran (1999), both absorption and emission features have been reported by various experiments prior to BATSE. Absorption lines in the 20-40 keV range were observed by several experiments. GINGA discovered several cases of lines with harmonic structure (Murakami et al. 1988; Fenimore et al. 1988). These lines were interpreted as cyclotron lines (reflecting a magnetic field of $\approx 10^{12}$ Gauss). Emission features near $400 \mathrm{keV}$ were claimed in other bursts (Mazets et al. 1980). However, so far, 
BATSE has not found any of the spectral features (absorption or emission lines) reported by earlier satellites (Palmer et al. 1994; Band et al. 1996). Meszaros \& Rees (1998) pointed out that within the relativistic fireball model an observed broadened spectral line might be a blue-shifted iron X-ray line. We suspect that, in detecting line features, the Doppler effect associated with the expanding fireball might play a role.

In the following we will investigate what one should expect in the spectrum if there are emission as well as absorption lines in the rest frame of fireballs.

\section{Effect on narrow emission lines}

We consider a fireball expanding with a definite value of Lorentz factor $\Gamma$ and study only the core content of the Doppler effect in the fireball framework with the cosmological as well as other effects being temporarily ignored. The rest frame radiation is assumed to be constant and independent of direction.

Let the fireball be observed at time $t$ and at frequency $v$. Then the expected spectrum would be (Paper I)

$v f_{v}(t)=\frac{2 \pi \widetilde{R}^{2}(t) v}{D^{2} \Gamma^{3}} \int_{0}^{\pi / 2} \frac{I_{0, v}\left(t_{0, \theta}, v_{0, \theta}\right) \cos \theta \sin \theta}{(1-\beta \cos \theta)^{5}} \mathrm{~d} \theta$,

with

$\widetilde{R}(t)=\beta\left[c\left(t-t_{\mathrm{c}}\right)-D\right]+R_{\mathrm{c}}$,

where $D$ is the distance of the fireball to the observer; $\theta$ is the angle to the line of sight; $v_{0, \theta}$ is the rest frame emission frequency of the differential surface, of the fireball, with $\theta ; t_{0, \theta}$ is the proper emission time of the differential surface; $t_{\mathrm{c}}$ is any coordinate time concerned; $R_{\mathrm{c}}$ is the radius of the fireball at time $t_{\mathrm{c}}$; and $I_{0, v}\left(t_{0, \theta}, v_{0, \theta}\right)$ is the rest frame intensity of the differential surface. Frequencies $v_{0, \theta}$ and $v$ are related by the Doppler effect. The proper time $t_{0, \theta}$ and observation time $t$ can be well linked by considering the travelling of light from the fireball to the observer (see Paper I).

Assume that during some period the radiation of the fireball is dominated by a certain mechanism and within this interval of time the rest frame radiation intensity can be expressed as:

$I_{0, v}\left(t_{0, \theta}, v_{0, \theta}\right)=I_{0}\left(t_{0, \theta}\right) g_{0, v}\left(v_{0, \theta}\right)$

where $g_{0, v}\left(v_{0, \theta}\right)$ describes the dominant radiation mechanism while $I_{0}\left(t_{0, \theta}\right)$ represents the development of the intensity magnitude. For constant radiations, $I_{0}\left(t_{0, \theta}\right)=I_{0}$. In this case, the spectrum would be

$v f_{v}(t)=\frac{2 \pi I_{0} \widetilde{R}^{2}(t) v}{D^{2} \Gamma^{3}} \int_{0}^{\pi / 2} g_{0, v}\left(v_{0, \theta}\right) \frac{\cos \theta \sin \theta}{(1-\beta \cos \theta)^{5}} \mathrm{~d} \theta$,

here the emission is assumed to cover the whole energy range.

As mentioned above, there is no single mechanism proposed that can well represent all the observed spectra of GRBs. In practice, an empirical form called the GRB model (Band et al. 1993) was frequently, and rather successfully, employed

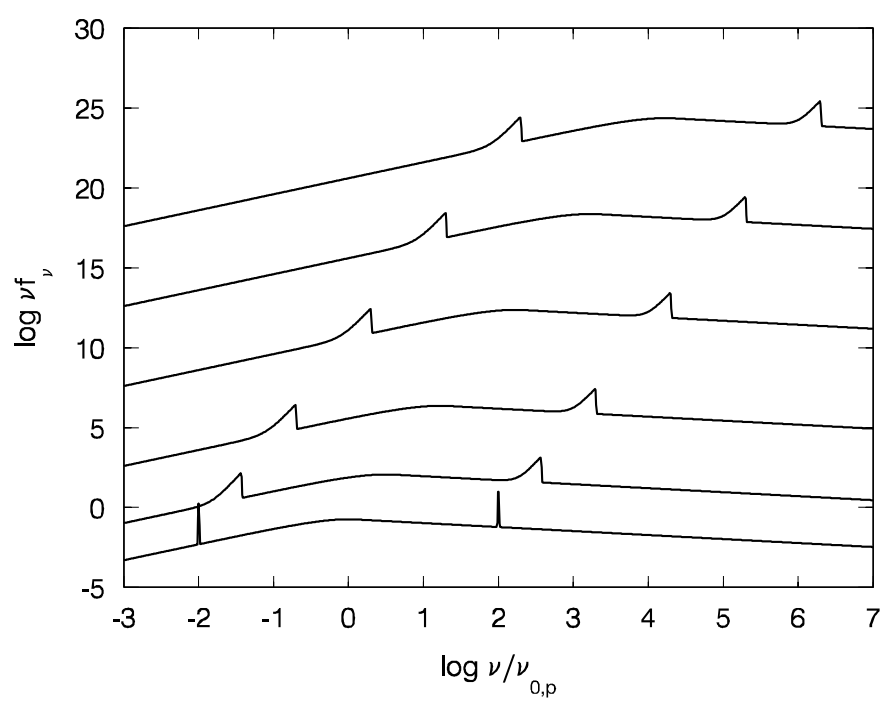

Fig. 1. The expected spectrum of a fireball with its rest frame radiation being the GRB form of $\alpha_{0}=-1$ and $\beta_{0}=-2.25$ plus the two emission lines defined by (8) and (9), observed at time $t$, where we take $2 \pi I_{0} v_{0, \mathrm{p}} \widetilde{R}^{2}(t) / D^{2}=1$. The solid lines from the bottom to the top correspond to $\Gamma=1,2,10,100,1000,10000$, respectively.

to fit most burst spectra (Ford et al. 1995; Preece et al. 2000). The relative intensity of the GRB model is (Band et al. 1993)

$g_{0, v, \mathrm{G}}\left(v_{0, \theta}\right)=\left\{\begin{array}{c}\left(\frac{v_{0, \theta}}{v_{0, \mathrm{p}}}\right)^{1+\alpha_{0, \mathrm{G}}} \exp \left[-\left(2+\alpha_{0, \mathrm{G}}\right) \frac{v_{0, \theta}}{v_{0, \mathrm{p}}}\right] \\ \left(\frac{v_{0, \theta}}{v_{0, \mathrm{p}}}<\frac{\alpha_{0, \mathrm{G}}-\beta_{0, \mathrm{G}}}{2+\alpha_{0, \mathrm{G}}}\right) \\ \left(\frac{\alpha_{0, \mathrm{G}}-\beta_{0, \mathrm{G}}}{2+\alpha_{0, \mathrm{G}}}\right)^{\alpha_{0, \mathrm{G}}-\beta_{0, \mathrm{G}}} \exp \left(\beta_{0, \mathrm{G}}-\alpha_{0, \mathrm{G}}\right)\left(\frac{v_{0, \theta}}{v_{0, \mathrm{p}}}\right)^{1+\beta_{0, \mathrm{G}}} \\ \left(\frac{v_{0, \theta}}{v_{0, \mathrm{p}}} \geq \frac{\alpha_{0, \mathrm{G}}-\beta_{0, \mathrm{G}}}{2+\alpha_{0, \mathrm{G}}}\right),\end{array}\right.$

where $\alpha_{0, \mathrm{G}}$ and $\beta_{0, \mathrm{G}}$ are the lower and higher indexes, respectively. As an illustration, we shall adopt in the following the GRB form with the typical values of the parameters: $\alpha_{0, \mathrm{G}}=-1$ and $\beta_{0, \mathrm{G}}=-2.25$ (Preece et al. 1998, 2000).

To show the effect on both emission and absorption lines, we consider the following rest frame radiation:

$g_{0, v}\left(v_{0, \theta}\right)=\max \left\{g_{0, v, \mathrm{G}}\left(v_{0, \theta}\right)+\sum_{i=1}^{n} e_{0, v, i}\left(v_{0, \theta}\right), 0\right\}$

where $e_{0, v, i}\left(v_{0, \theta}\right)$ describes the relative intensity of lines:

$$
\begin{aligned}
e_{0, v, i}\left(v_{0, \theta}\right)= & \frac{h_{i}}{\exp \left(2+\alpha_{0}\right)} \\
& \times \exp \left[-\frac{\left(v_{0, \theta} / v_{0, \mathrm{p}}-v_{0, \text { line }, i} / v_{0, \mathrm{p}}\right)^{2}}{2 \times 10^{n_{i}}}\right] .
\end{aligned}
$$

Now we consider two narrow emission lines and take

$v_{0, \text { line }, 1} / v_{0, \mathrm{p}}=0.01, \quad h_{1}=1000, \quad n_{1}=-8$

and

$v_{0, \text { line }, 2} / v_{0, \mathrm{p}}=100, \quad h_{2}=1, \quad n_{2}=0$.

Shown in Fig. 1 are the $\log \left(v f_{v}\right)-\log \left(v / v_{0, \mathrm{p}}\right)$ curves of the fireball containing in its rest frame radiation the two emission 
Table 1. List of $v_{\text {line }}$ and $\Delta v_{F W H M} / v_{\text {line }}$ for the two narrow emission lines.

\begin{tabular}{lllll}
\hline \hline & \multicolumn{2}{l}{$v_{0, \text { line }} / v_{0, \mathrm{p}}=0.01$} & \multicolumn{2}{l}{$v_{0, \text { line }} / v_{0, \mathrm{p}}=100$} \\
\hline$\Gamma$ & $v_{\text {line }} / v_{0, \mathrm{p}}$ & $\Delta v_{F W H M} / v_{\text {line }}$ & $v_{\text {line }} / v_{0, \mathrm{p}}$ & $\Delta v_{F W H M} / v_{\text {line }}$ \\
\hline $1 \times 10^{0}$ & $1.00 \times 10^{-2}$ & $2.35 \times 10^{-2}$ & $1.00 \times 10^{2}$ & $2.35 \times 10^{-2}$ \\
$2 \times 10^{0}$ & $3.65 \times 10^{-2}$ & $1.79 \times 10^{-1}$ & $3.65 \times 10^{2}$ & $1.79 \times 10^{-1}$ \\
$1 \times 10^{1}$ & $1.95 \times 10^{-1}$ & $1.86 \times 10^{-1}$ & $1.95 \times 10^{3}$ & $1.86 \times 10^{-1}$ \\
$1 \times 10^{2}$ & $1.96 \times 10^{0}$ & $1.86 \times 10^{-1}$ & $1.96 \times 10^{4}$ & $1.86 \times 10^{-1}$ \\
$1 \times 10^{3}$ & $1.96 \times 10^{1}$ & $1.86 \times 10^{-1}$ & $1.96 \times 10^{5}$ & $1.86 \times 10^{-1}$ \\
$1 \times 10^{4}$ & $1.96 \times 10^{2}$ & $1.86 \times 10^{-1}$ & $1.96 \times 10^{6}$ & $1.86 \times 10^{-1}$ \\
\hline
\end{tabular}

lines, for various values of $\Gamma$. The figure shows that, in the spectrum of a relativistically expanding fireball, rest frame narrow emission lines indeed shift to higher energy bands and are significantly broadened. Therefore, there would be no narrow emission lines expected in the spectrum of the object. In addition, a rest frame narrow emission line at high energy bands would form an up-turning tip of the high energy tail in the observed spectrum (an observed $\mathrm{MeV}$ tip can come from a rest frame $\mathrm{keV}$ line when $\Gamma=1000$ or from a $10 \mathrm{keV}$ line when $\Gamma=100)$ (see Fig. 1).

To show the effect of the broadening, we calculate the relative width of the frequency range related to the $F W H M$ of the line feature, $\Delta v_{F W H M} / v_{\text {line }}$, where $v_{\text {line }}$ is the observed line frequency at which the peak flux of the line feature is found. Listed in Table 1 are the values of $v_{\text {line }}$ as well as $\Delta v_{F W H M} / v_{\text {line }}$, coupled to various values of $\Gamma$, for the two lines.

We find that $v_{\text {line }}$ is a linear function of $\Gamma$, approximately following $v_{\text {line }} \approx 2 \Gamma v_{0 \text {,line }}$, when $\Gamma$ is large enough (say, $\Gamma>10$ ). Therefore, if $v_{\text {line }}$ is identified, then one can take it as an indicator of the expansion speed of the fireball. It also shows that, in the case discussed above, where the relative width of the rest frame narrow emission lines is 0.0235 (see the case of $\Gamma=1$ in Table 1), the relative width of the line feature becomes almost one magnitude larger than that of the rest frame emission line when the fireball expands relativistically. When $\Gamma$ is large enough, the relative width would approach an asymptotic value (here it is 0.186).

As illustrated by an analytical calculation (see Appendix A), $v_{\text {line }}$ of a very narrow rectangle emission, which is taken to represent a very narrow emission line, can be determined by $v_{\text {line }}=v_{0, \text { line }} / \Gamma(1-\beta)$. When $\Gamma \gg 1$, it approaches $2 \Gamma v_{0, \text { line }}$, which is the same as that shown in Table 1. In this situation, the value of $\Delta v_{F W H M} / v_{\text {line }}$ can be estimated by $0.162+0.415 \Delta v_{0, \text { line }} / v_{0, \text { line }}$. When $\Delta v_{0, \text { line }} / v_{0 \text {, line }} \rightarrow 0$, $\Delta v_{F W H M} / v_{\text {line }} \simeq 0.162$. The corresponding value shown in Table 1 is slightly larger than this. It might be due to the width of the rest frame line feature. When calculating the line described by (9) in the case of $\Gamma=100$ by replacing $n_{2}=0$ with $n_{2}=-2$, we really obtain $\Delta v_{F W H M} / v_{\text {line }} \simeq 0.162$.

\section{Effect on other lines}

We consider in the following the effect on other lines.

\section{a) Weak narrow emission lines}

We take

$v_{0, \text { line }, 1} / v_{0, \mathrm{p}}=0.01, \quad h_{1}=10, \quad n_{1}=-8$

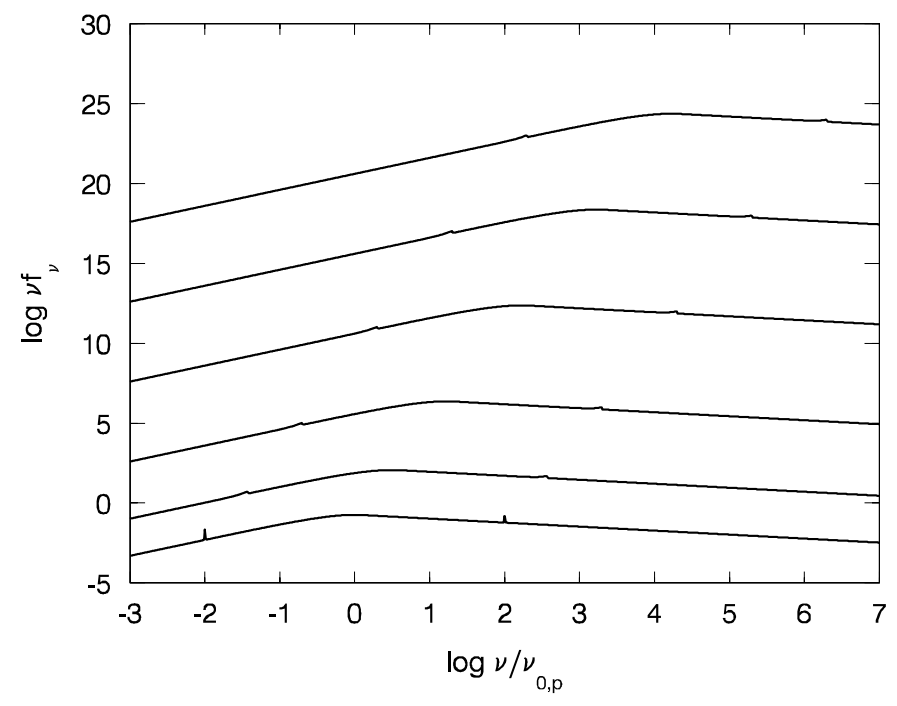

Fig. 2. The expected spectrum of a fireball with its rest frame radiation containing the two weak emission lines defined by (10) and (11), where other parameters and the symbols are the same as those adopted in Fig. 1.

and

$v_{0, \text { line }, 2} / v_{0, \mathrm{p}}=100, \quad h_{2}=0.01, \quad n_{2}=0$.

Shown in Fig. 2 are the $\log \left(v f_{v}\right)-\log \left(v / v_{0, \mathrm{p}}\right)$ curves with the two weak emission lines. The figure shows that, in the spectrum of the fireball considered above, rest frame weak narrow emission lines also shift to higher energy bands but are significantly smoothed and would hardly be detectable.

\section{b) Broad emission lines}

We take

$v_{0, \text { line }, 1} / v_{0, \mathrm{p}}=0.01, \quad h_{1}=10, \quad n_{1}=-5$

and

$v_{0, \text { line }, 2} / v_{0, \mathrm{p}}=100, \quad h_{2}=0.01, \quad n_{2}=3$.

Shown in Fig. 3 are the $\log \left(v f_{v}\right)-\log \left(v / v_{0, \mathrm{p}}\right)$ curves with the two broad emission lines. It shows that rest frame broad emission lines also shift to higher energy bands but their features remain almost unchanged and therefore would be easier to detect.

c) Narrow absorption lines

We take

$v_{0, \text { line }, 1} / v_{0, \mathrm{p}}=0.01, \quad h_{1}=-1000, \quad n_{1}=-8$ 


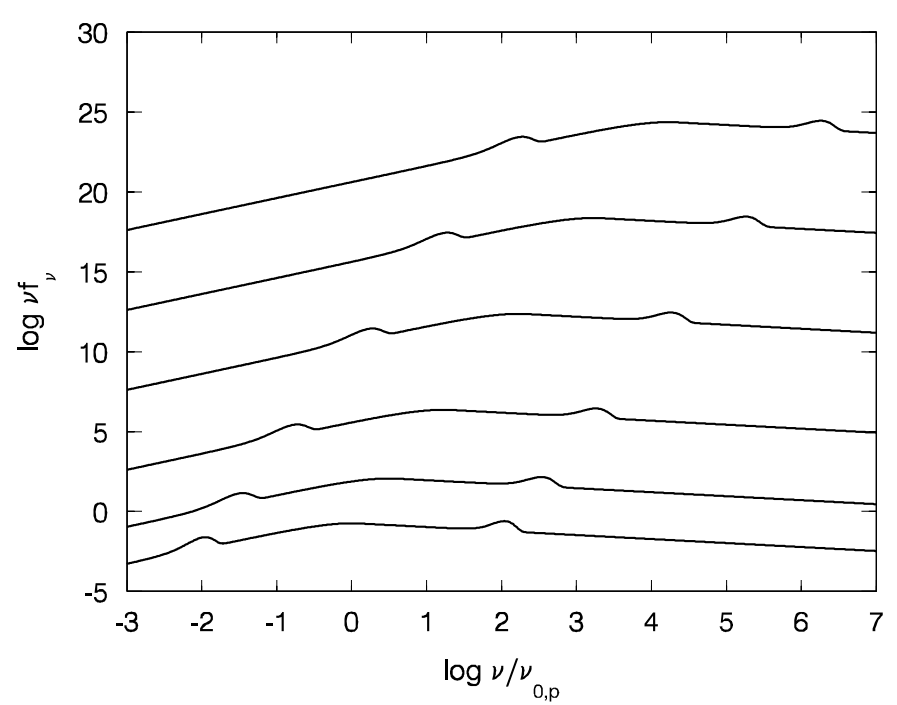

Fig. 3. The expected spectrum of a fireball with its rest frame radiation containing the two broad emission lines defined by (12) and (13), where other parameters and the symbols are the same as those adopted in Fig. 1.

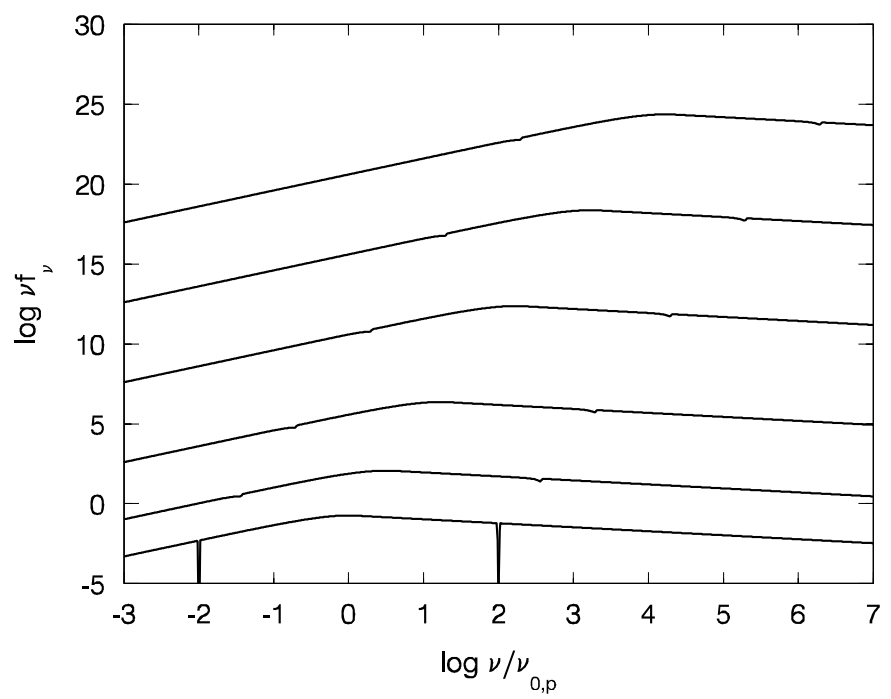

Fig. 4. The expected spectrum of a fireball with its rest frame radiation containing the two narrow absorption lines defined by (14) and (15), where other parameters and the symbols are the same as those adopted in Fig. 1.

and

$v_{0, \text { line }, 2} / v_{0, \mathrm{p}}=100, \quad h_{2}=-1, \quad n_{2}=0$.

Shown in Fig. 4 are the $\log \left(v f_{v}\right)-\log \left(v / v_{0, \mathrm{p}}\right)$ curves with the two narrow absorption lines. It shows that rest frame narrow absorption lines also shift to higher energy bands but are significantly smoothed and would hardly be detectable.

\section{d) Broad absorption lines}

We take

$v_{0, \text { line }, 1} / v_{0, \mathrm{p}}=0.01, \quad h_{1}=-10, \quad n_{1}=-5$

and

$v_{0, \text { line }, 2} / v_{0, \mathrm{p}}=100, \quad h_{2}=-0.01, \quad n_{2}=3$.

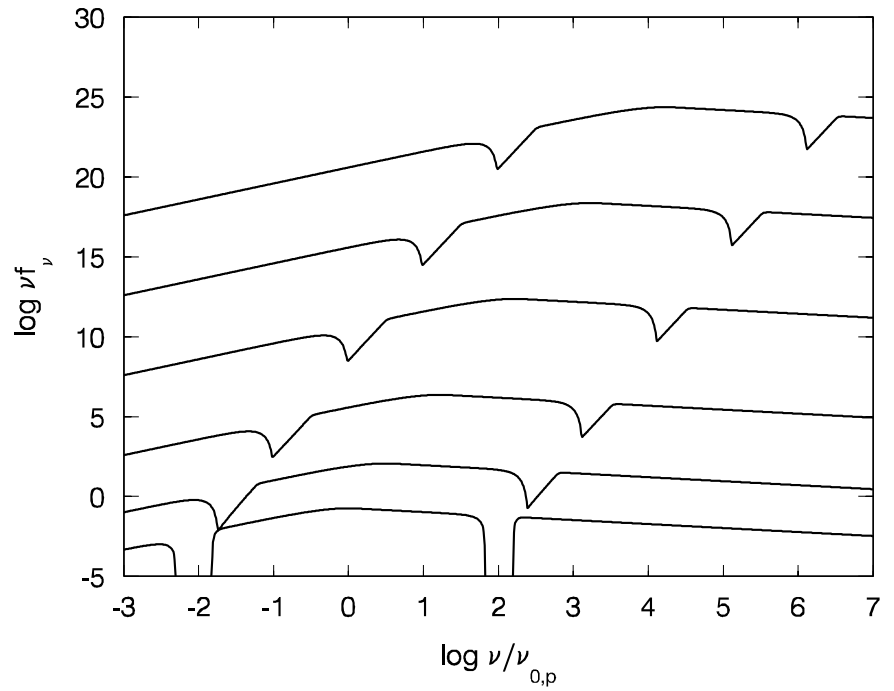

Fig. 5. The expected spectrum of a fireball with its rest frame radiation containing the two broad absorption lines defined by (16) and (17), where other parameters and the symbols are the same as those adopted in Fig. 1.

Shown in Fig. 5 are the $\log \left(v f_{v}\right)-\log \left(v / v_{0, \mathrm{p}}\right)$ curves with the two broad absorption lines. It shows that rest frame broad absorption lines also shift to higher energy bands but are significantly smoothed so that deep gaps would be significantly filled. The broad absorption features would remain and therefore would be detectable.

\section{e) Weak broad absorption lines}

We take

$v_{0, \text { line }, 1} / v_{0, \mathrm{p}}=0.01, \quad h_{1}=-2, \quad n_{1}=-5$

and

$v_{0, \text { line }, 2} / v_{0, \mathrm{p}}=100, \quad h_{2}=-0.002, \quad n_{2}=3$.

Shown in Fig. 6 are the $\log \left(v f_{v}\right)-\log \left(v / v_{0, \mathrm{p}}\right)$ curves with the two weak broad absorption lines. It shows that rest frame weak broad absorption lines also shift to higher energy bands but their features remain almost unchanged and would be detectable.

f) Emission line forest

We consider two emission line forests at lower and higher energy bands respectively. We take

$\begin{array}{lll}v_{0, \text { line }, 1} / v_{0, \mathrm{p}}=0.01, & h_{1}=1000, & n_{1}=-8, \\ v_{0, \text { line }, 2} / v_{0, \mathrm{p}}=0.012, & h_{2}=1000, & n_{2}=-8, \\ v_{0, \text { line }, 3} / v_{0, \mathrm{p}}=0.014, & h_{3}=1000, & n_{3}=-8, \\ v_{0, \text { line }, 4} / v_{0, \mathrm{p}}=0.016, & h_{4}=1000, & n_{4}=-8,\end{array}$

and

$v_{0, \text { line }, 5} / v_{0, \mathrm{p}}=100, \quad h_{5}=1, \quad n_{5}=0$,

$v_{0, \text { line }, 6} / v_{0, \mathrm{p}}=120, \quad h_{6}=1, \quad n_{6}=0$,

$v_{0, \text { line }, 7} / v_{0, \mathrm{p}}=140, \quad h_{7}=1, \quad n_{7}=0$, 


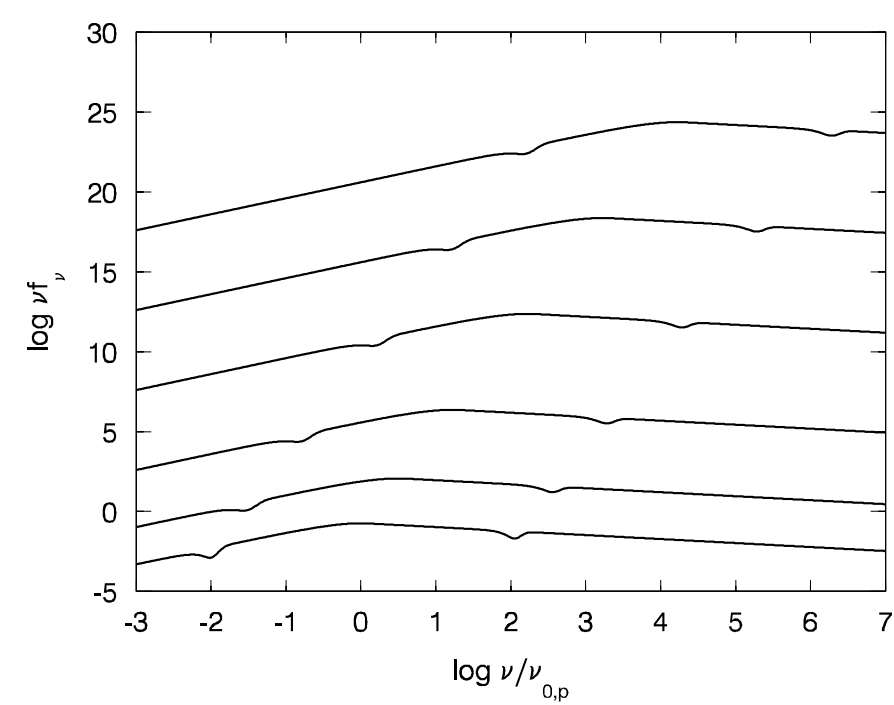

Fig. 6. The expected spectrum of a fireball with its rest frame radiation containing the two weak broad absorption lines defined by (18) and (19), where other parameters and the symbols are the same as those adopted in Fig. 1.

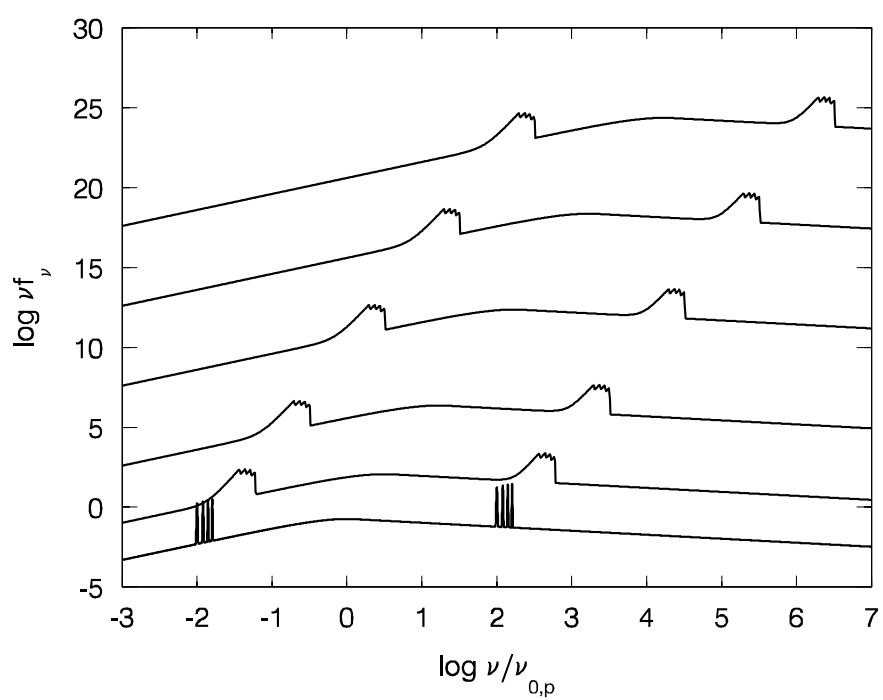

Fig. 7. The expected spectrum of a fireball with its rest frame radiation containing the two emission line forests defined by (20)-(27), where other parameters and the symbols are the same as those adopted in Fig. 1.

$v_{0, \text { line }, 8} / v_{0, \mathrm{p}}=160, \quad h_{8}=1, \quad n_{8}=0$.

Shown in Fig. 7 are the $\log \left(v f_{v}\right)-\log \left(v / v_{0, \mathrm{p}}\right)$ curves with the two emission line forests. It shows that rest frame emission line forests also shift to higher energy bands but they are significantly smoothed and each of them forms a single broad line feature.

\section{g) Absorption line forest}

We consider two absorption line forests at lower and higher energy bands respectively and take

$$
\begin{array}{lll}
v_{0, \text { line }, 1} / v_{0, \mathrm{p}}=0.01, & h_{1}=-1000, & n_{1}=-8, \\
v_{0, \text { line }, 2} / v_{0, \mathrm{p}}=0.012, & h_{2}=-1000, & n_{2}=-8, \\
v_{0, \text { line }, 3} / v_{0, \mathrm{p}}=0.014, & h_{3}=-1000, & n_{3}=-8,
\end{array}
$$

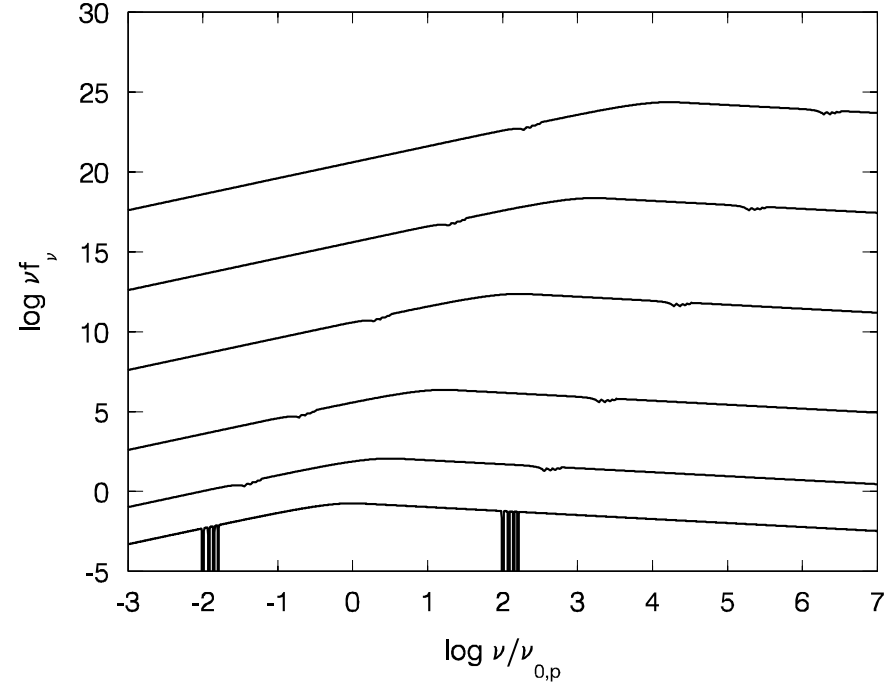

Fig. 8. The expected spectrum of a fireball with its rest frame radiation containing the two absorption line forests defined by (28)-(35), where other parameters and the symbols are the same as those adopted in Fig. 1.

$v_{0, \text { line }, 4} / v_{0, \mathrm{p}}=0.016, \quad h_{4}=-1000, \quad n_{4}=-8$,

and

$v_{0, \text { line }, 5} / v_{0, \mathrm{p}}=100, \quad h_{5}=-1, \quad n_{5}=0$,

$v_{0, \text { line }, 6} / v_{0, \mathrm{p}}=120, \quad h_{6}=-1, \quad n_{6}=0$,

$v_{0, \text { line }, 7} / v_{0, \mathrm{p}}=140, \quad h_{7}=-1, \quad n_{7}=0$

$v_{0, \text { line }, 8} / v_{0, \mathrm{p}}=160, \quad h_{8}=-1, \quad n_{8}=0$.

Shown in Fig. 8 are the $\log \left(v f_{v}\right)-\log \left(v / v_{0, p}\right)$ curves with the two absorption line forests. It shows that rest frame absorption line forests also shift to higher energy bands but are significantly smoothed and would hardly be detectable.

\section{Effect of time dependence of the line intensity or variation of the Lorentz factor}

Here we investigate the effect of time dependence of the line intensity and the effect of variation of $\Gamma$.

a) The case of the line intensity varying with time

We consider rest frame radiation containing a constant GRB form and two time dependent intensity narrow lines. In this situation, the formulas adopted in Sect. 2 are valid when we replace $h_{i}$ in (7) with $h_{i}\left(t_{0, \theta}\right)$. Suppose the line intensity is an exponential function of time:

$h_{i}\left(t_{0, \theta}\right)=h_{i, 0} \exp \left(-\frac{t_{0, \theta}-t_{0, \mathrm{c}}}{\tau_{0}}\right) \quad\left(t_{0, \theta} \geq t_{0, \mathrm{c}}\right)$,

where $h_{i, 0}, t_{0, \mathrm{c}}$ and $\tau_{0}$ are constants. As shown in Paper I, $t_{0, \theta}$ and $t$ can be related by

$t_{0, \theta}=\frac{t-t_{\mathrm{c}}-D / c+\left(R_{\mathrm{c}} / c\right) \cos \theta}{\Gamma(1-\beta \cos \theta)}+t_{0, \mathrm{c}}$ 


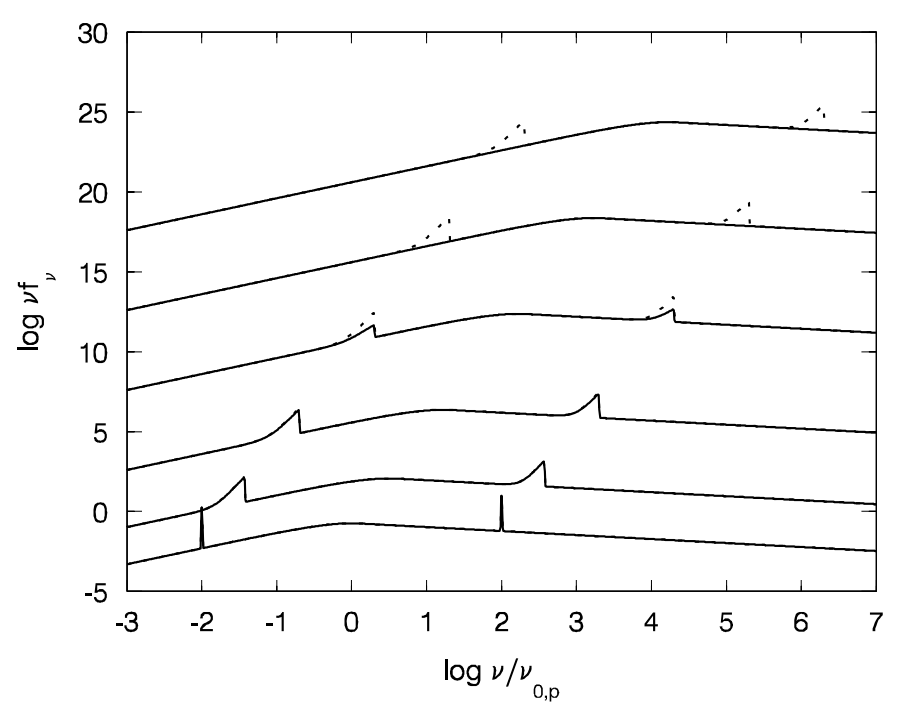

Fig. 9. The expected spectrum of a fireball containing in its rest frame radiation the two time dependent intensity emission lines [confined by (39)] for observation time $t_{1}$ [defined by (40)], where we take $2 \pi I_{0} v_{0, \mathrm{p}} \widetilde{R}^{2}\left(t_{1}\right) / D^{2}=1$. The solid lines from the bottom to the top correspond to $\Gamma=1,2,10,100,1000,10000$, respectively, and the dotted lines (some are totally overlapped by the corresponding solid lines) are those presented in Fig. 1.

where $t_{\mathrm{c}}$ and $R_{\mathrm{c}}$ are constants. We take

$\tau_{0}=100 R_{\mathrm{c}} / c$.

Therefore,

$$
\begin{aligned}
h_{i}\left(t_{0, \theta}\right)= & h_{i, 0} \exp \left[-\frac{\frac{c\left(t-t_{\mathrm{c}}\right)-D}{R_{\mathrm{c}}}+\cos \theta}{100 \Gamma(1-\beta \cos \theta)}\right] \\
& {\left[\frac{t-t_{\mathrm{c}}-D / c+\left(R_{\mathrm{c}} / c\right) \cos \theta}{\Gamma(1-\beta \cos \theta)} \geq 0\right] . }
\end{aligned}
$$

We consider different observation times and take

$t_{1}=\frac{D}{c}+t_{\mathrm{c}}$,

$t_{2}=\frac{R_{\mathrm{c}}+D}{c}+t_{\mathrm{c}}$

and

$t_{3}=\frac{10 R_{\mathrm{c}}+D}{c}+t_{\mathrm{c}}$.

Once more we adopt (8) and (9), where $h_{i}$ stands for $h_{i, 0}(i=$ $1,2)$.

The plots of $\log \left(v f_{v}\right)-\log \left(v / v_{0, \mathrm{p}}\right)$ for the fireball containing in its rest frame radiation the two time dependent intensity emission lines for the three observation times are shown by Figs. 9-11 respectively, where various values of $\Gamma$ are considered for each figure. For the sake of comparison, we take $2 \pi I_{0} v_{0, \mathrm{p}} \widetilde{R}^{2}\left(t_{1}\right) / D^{2}=1,2 \pi I_{0} v_{0, \mathrm{p}} \widetilde{R}^{2}\left(t_{2}\right) / D^{2}=1$ and $2 \pi I_{0} v_{0, \mathrm{p}} \widetilde{R}^{2}\left(t_{3}\right) / D^{2}=1$ for the three figures, and the curves shown in Fig. 1. are also plotted.

Similar to that shown in Fig. 1, in the spectrum of a relativistically expanding fireball, rest frame dimming narrow

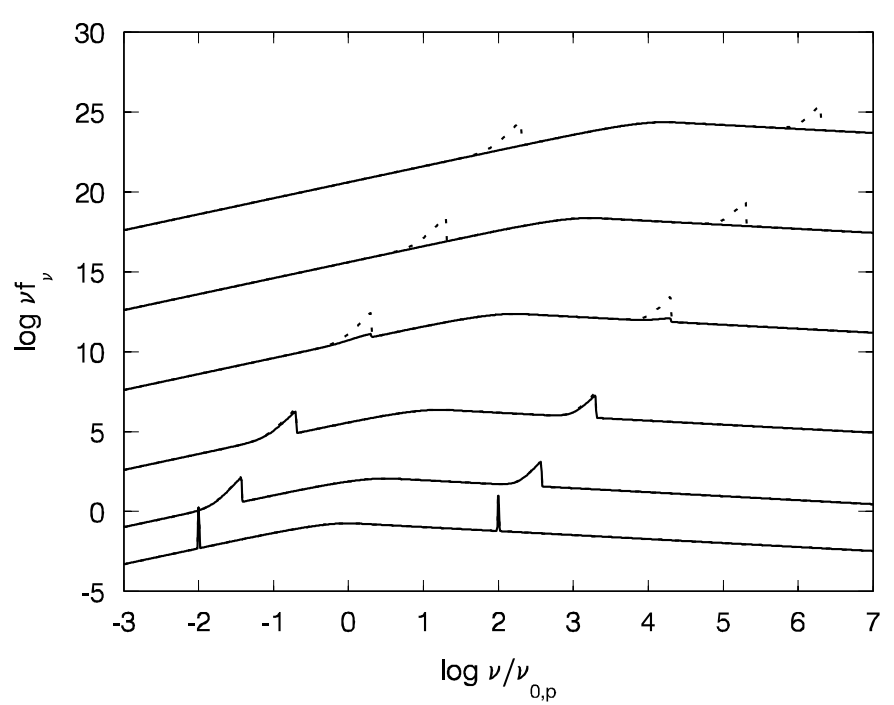

Fig. 10. The expected spectrum of a fireball containing in its rest frame radiation the two time dependent intensity emission lines for observation time $t_{2}$ [defined by (41)], where we take $2 \pi I_{0} v_{0, \mathrm{p}} \widetilde{R}^{2}\left(t_{2}\right) / D^{2}=1$. Other parameters and the symbols are the same as those adopted in Fig. 9.

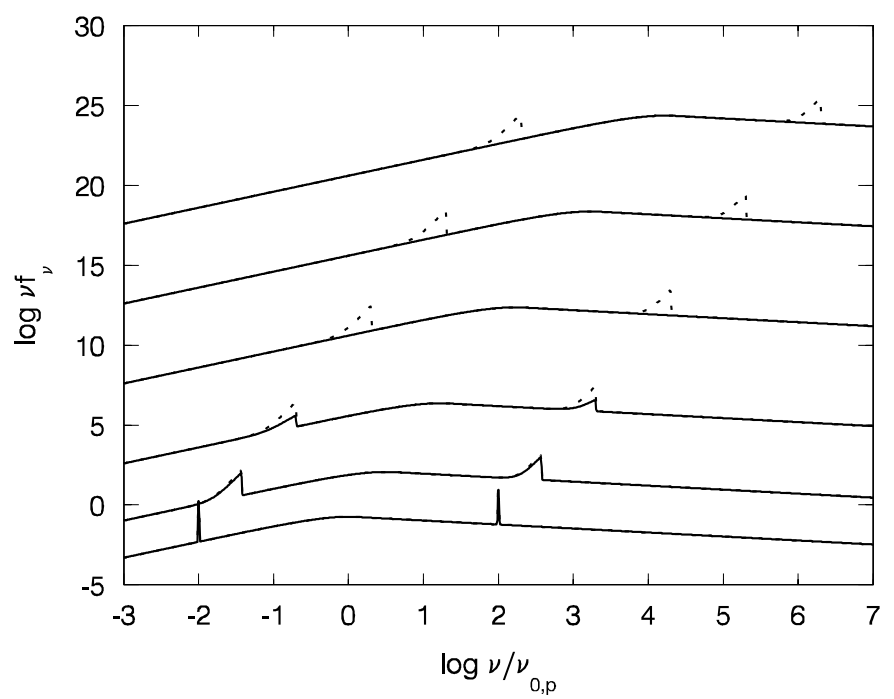

Fig. 11. The expected spectrum of a fireball containing in its rest frame radiation the two time dependent intensity emission lines for observation time $t_{3}$ [defined by (42)], where we take $2 \pi I_{0} v_{0, \mathrm{p}} \widetilde{R}^{2}\left(t_{3}\right) / D^{2}=1$. Other parameters and the symbols are the same as those adopted in Fig. 9.

emission lines would also shift to higher energy bands and would be significantly broadened. However, for fireballs with a very large Lorentz factor (say, $\Gamma>1000$ ), the line feature would disappear. As time progresses, the feature would eventually disappear and this would happen at a much earlier time for those fireballs with a relatively larger value of the Lorentz factor.

\section{b) The case of variation of the Lorentz factor}

Here we study the effect of variation of the Lorentz factor on the spectrum of fireballs. The expected spectrum of fireballs influenced by this variation is studied in Appendix B. The effect would be shown more clearly when the influenced 
spectrum is compared with the previously discussed one. Therefore, we consider the same rest frame constant radiation discussed in Sect. 2, with the variation of the Lorentz factor being the only difference. To employ what we derived in Appendix $\mathrm{B}$, we assume that the radiation is independent of direction, $I_{0, v}\left(t_{0, \theta}, v_{0, \theta}, \theta\right)=I_{0, v}\left(t_{0, \theta}, v_{0, \theta}\right)$, and there is no limit for the radiated frequency and the radiation concerned lasts a sufficiently large interval of time so that the integral limits of (B.15) are $\theta_{\min }=0$ and $\theta_{\max }=\pi / 2$ (see Paper I for a detailed discussion). Applying (3), (B.7) and (B.16), we obtain from (B.15) that

$$
\begin{aligned}
v f_{v}(t)= & \frac{2 \pi I_{0} v}{D^{2}} \int_{0}^{\pi / 2} \frac{R^{2}\left(t_{\theta}\right) g_{0, v}\left(v_{0, \theta}\right)}{\Gamma^{3}\left(t_{\theta}\right)\left[1-\beta\left(t_{\theta}\right) \cos \theta\right]^{3}} \\
& \times \cos \theta \sin \theta \mathrm{d} \theta,
\end{aligned}
$$

where we take $I_{0}\left(t_{0, \theta}\right)=I_{0}$, and $g_{0, v}\left(v_{0, \theta}\right)$ is determined by (6). Frequencies $v_{0, \theta}$ and $v$ are related by the Doppler effect (see (B.7)).

It is known that, in its deceleration phase, the Lorentz factor of a fireball would develop with time following $t^{-3 / 8}$ in the case of the adiabatic hydrodynamics while following $t^{-3 / 7}$ in the case of the radiative hydrodynamics (Piran 1999). To illustrate the effect, we consider the latter case and assume

$\Gamma\left(t_{\theta}\right)=\max \left\{k t_{\theta}^{-3 / 7}, 1\right\} \quad\left(t_{\theta} \geq t_{\mathrm{c}}\right)$,

where $k$ is a constant. Here we assign $t_{\mathrm{c}}$ to be the time when the deceleration starts and assign $\Gamma=1$ when the deceleration as well as the expansion stop. Suppose $\Gamma_{\mathrm{c}}>1$ is the value of the Lorentz factor of the fireball at time $t_{\mathrm{c}}$, then one obtains

$k=\Gamma_{\mathrm{c}} t_{\mathrm{c}}^{3 / 7}$.

When $\Gamma\left(t_{\theta}\right)$ is known, the relation between the coordinate time, $t_{\theta}$, of the differential surface of the fireball concerned and the observation time, $t$, will be well established, and the radius of the fireball as a function of time will be determined (see Appendix B). From (B.9) we get

$$
\begin{array}{r}
t_{\theta}-\left[\int_{t_{\mathrm{c}}}^{t_{\theta}} \sqrt{1-\frac{1}{\left(\max \left\{k t_{\theta}^{-3 / 7}, 1\right\}\right)^{2}}} \mathrm{~d} t_{\theta}\right] \\
\times \cos \theta=t+\frac{R_{\mathrm{c}}}{c} \cos \theta-\frac{D}{c} .
\end{array}
$$

We find that, once $t$ is assigned, $t_{\theta}$ will be determined by (46). Then $\Gamma\left(t_{\theta}\right)$ will be determined by (44). Once both $t$ and $t_{\theta}$ are known, $R\left(t_{\theta}\right)$ will be easily determined. We get from (B.8) and (46) that

$$
\begin{aligned}
R\left(t_{\theta}\right) & =\frac{D-c\left(t-t_{\theta}\right)}{\cos \theta} \\
& =R_{\mathrm{c}}+c \int_{t_{\mathrm{c}}}^{t_{\theta}} \sqrt{1-\frac{1}{\left(\max \left\{k t_{\theta}^{-3 / 7}, 1\right\}\right)^{2}}} \mathrm{~d} t_{\theta} .
\end{aligned}
$$

To show the effect, different observation times should be concerned. Let

$p \equiv \frac{c\left(t-t_{\mathrm{c}}\right)-D}{R_{\mathrm{c}}}$.
Then

$t=t_{\mathrm{c}}+\frac{D+p R_{\mathrm{c}}}{c}$

We then come to

$$
\begin{array}{r}
t_{\theta}-\left[\int_{t_{\mathrm{c}}}^{t_{\theta}} \sqrt{1-\frac{1}{\left(\max \left\{k t_{\theta}^{-3 / 7}, 1\right\}\right)^{2}}} \mathrm{~d} t_{\theta}\right] \\
\times \cos \theta=t_{\mathrm{c}}+\frac{R_{\mathrm{c}}}{c}(p+\cos \theta)
\end{array}
$$

and

$$
R\left(t_{\theta}\right)=\frac{R_{\mathrm{c}}}{\cos \theta}\left(\frac{t_{\theta}-t_{\mathrm{c}}}{R_{\mathrm{c}} / c}-p\right) .
$$

To focus on the effect of variation of the Lorentz factor, we require that, all photons observed at the assigned time must be those emitted after the deceleration starts and before the expansion stops. The first requirement leads to $p \geq 0$ (see Appendix C). To meet the second requirement, $k$ cannot be arbitrarily taken as long as $p, \Gamma_{\mathrm{c}}$ and $R_{\mathrm{c}}$ are fixed. For given values of $p, \Gamma_{\mathrm{c}}$ and $R_{\mathrm{c}}$, there will a lower limit of $k$ (see Appendix C), i.e.,

$k>k_{0}$,

with

$k_{0} \equiv \Gamma_{\mathrm{c}}\left[\frac{R_{\mathrm{c}}(1+p)}{c\left(1-\beta_{\mathrm{c}}\right)\left(\Gamma_{\mathrm{c}}^{7 / 3}-1\right)}\right]^{3 / 7}$,

where $\beta_{\mathrm{c}} \equiv \sqrt{\Gamma_{\mathrm{c}}^{2}-1} / \Gamma_{\mathrm{c}}$.

To show the development of the spectrum, we consider two different observation times and take $p=(0,10)$. For the sake of comparison, we adopt $R_{\mathrm{c}} / c=1$ and take $2 \pi I_{0} v_{0, \mathrm{p}} R_{\mathrm{c}}^{2} / D^{2}=1$ and $k=10 k_{0}\left(p=10, \Gamma_{\mathrm{c}}, R_{\mathrm{c}}\right)$ for all situations concerned.

The results are displayed in Figs. 12-16. All these figures show that the flux observed at a relatively later time would be larger than that observed at an earlier time, suggesting that, in the situation studied here, the enhancement of the radius of the fireball plays an important role in the magnitude of the flux.

Shown in Fig. 12 we find that, when $\Gamma_{\mathrm{c}}$ is very small, as the variation of the Lorentz factor is then not obvious, the effect of deceleration would be insignificant. The positions of emission lines would remain almost unchanged.

Figures 14-16 reveal that the flux affected by the deceleration would become much smaller than the constant radiation flux when the initial Lorentz factor is large enough (say, $\Gamma_{\mathrm{c}} \geq 100$ ), and the change of the positions of emission lines due to the decrease of the Lorentz factor would be quite significant (the positions of the lines would shift to lower energy bands as time progresses).

We find in Fig. 14 an unfamiliar form of emission lines. To view this phenomenon in more detail, see Fig. 17, where more lines with different values of $\Gamma_{\mathrm{c}}$ are presented (a detailed study of this issue will be made later). It shows that, for the assigned observation time, the emission lines concerned would be sharp on both edges in some cases where the initial Lorentz factor is carefully chosen. To find out if this phenomenon depends also 


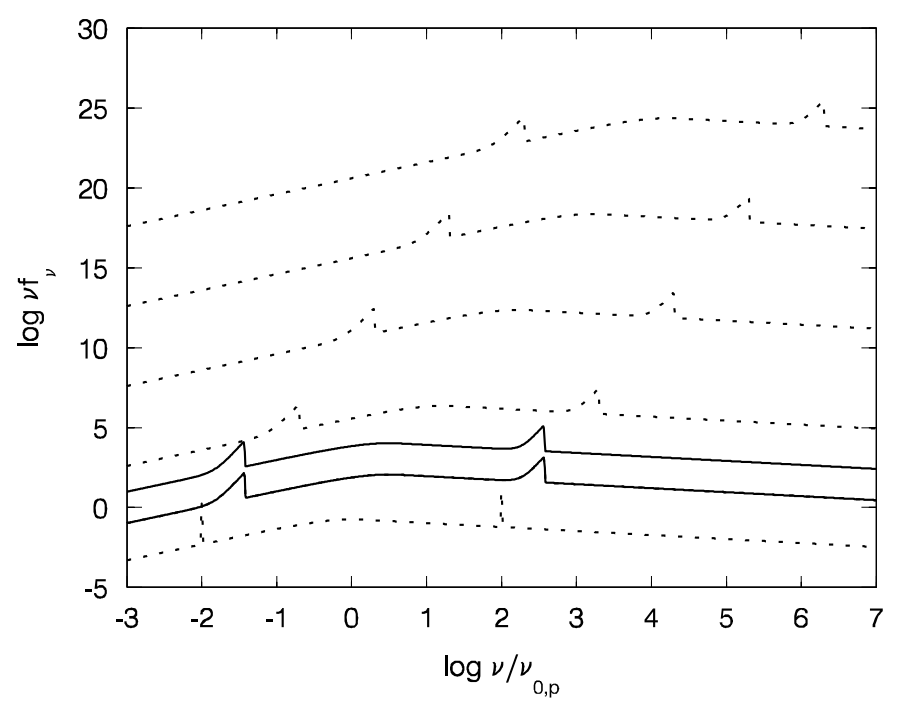

Fig. 12. The expected spectrum of a fireball with a variable Lorentz factor, where the initial Lorentz factor is $\Gamma_{\mathrm{c}}=2$ and the radiation concerned is that discussed in Sect. 2 . We take $R_{\mathrm{c}} / c=1,2 \pi I_{0} v_{0, \mathrm{p}} R_{\mathrm{c}}^{2} / D^{2}=$ 1 , and $k=10 k_{0}\left(p=10, \Gamma_{\mathrm{c}}=2, R_{\mathrm{c}} / c=1\right)$. The solid lines from the bottom to the top correspond to $p=0,10$, respectively, and the dotted lines (some are totally overlapped by the corresponding solid lines) are those presented in Fig. 1.

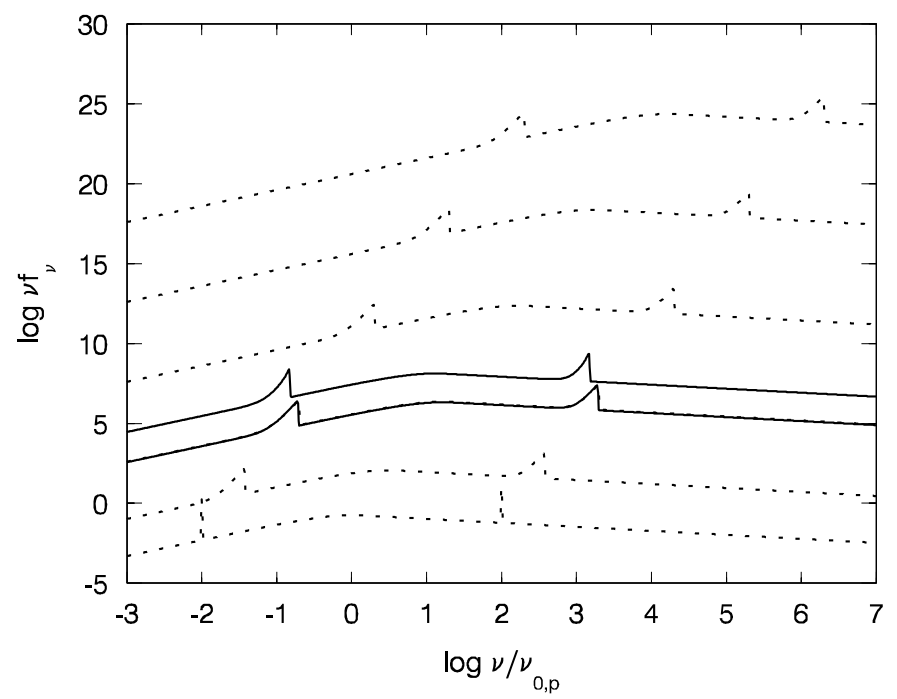

Fig. 13. The expected spectrum of a fireball with a variable Lorentz factor, where $\Gamma_{\mathrm{c}}=10$ and $k=10 k_{0}\left(p=10, \Gamma_{\mathrm{c}}=10, R_{\mathrm{c}} / c=1\right)$. Other parameters and the symbols are the same as those adopted in Fig. 12.

on the observation time, we plot Fig. 18, where different observation times are considered for a single initial Lorentz factor. One finds that the shape of the lines is indeed changed with time. During some period, it would be sharp on both edges. Meanwhile, the flux keeps being enhanced (at least during the epoch of the deceleration phase concerned). This would become an interesting expected observational characteristic.

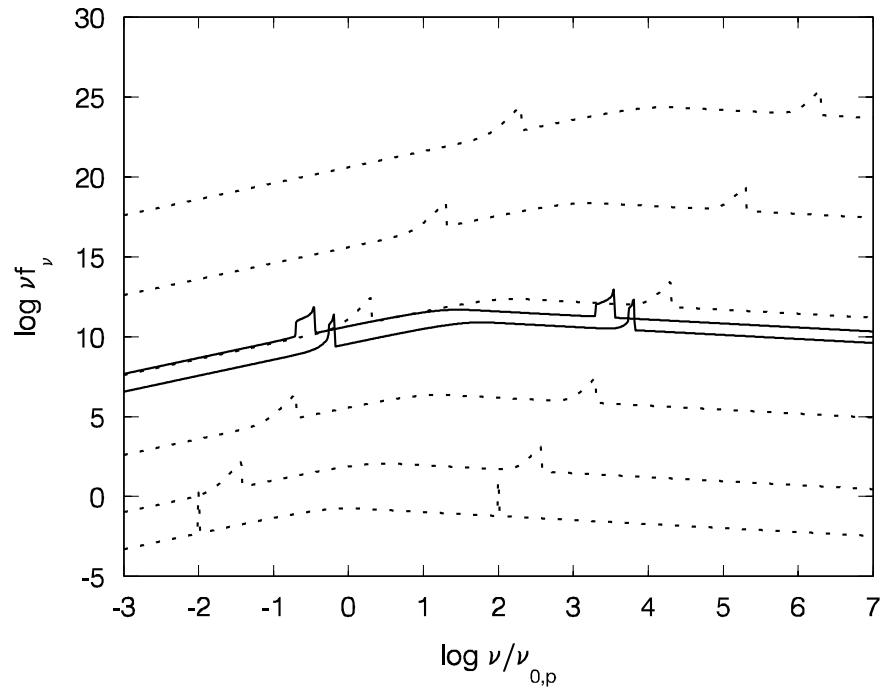

Fig. 14. The expected spectrum of a fireball with a variable Lorentz factor, where $\Gamma_{\mathrm{c}}=100$ and $k=10 k_{0}\left(p=10, \Gamma_{\mathrm{c}}=100, R_{\mathrm{c}} / c=1\right)$. Other parameters and the symbols are the same as those adopted in Fig. 12.

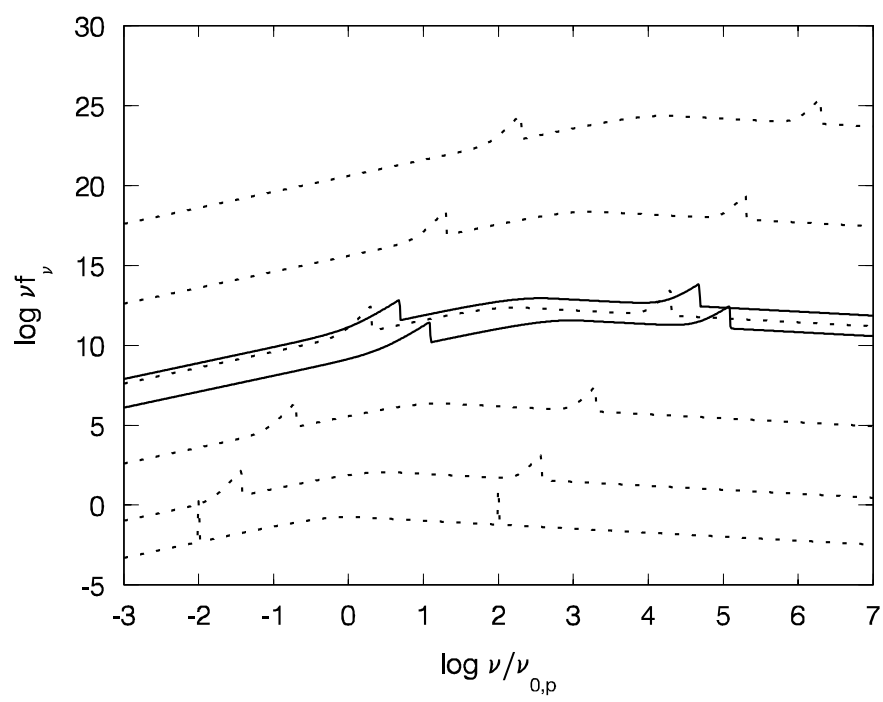

Fig. 15. The expected spectrum of a fireball with a variable Lorentz factor, where $\Gamma_{\mathrm{c}}=1000$ and $k=10 k_{0}\left(p=10, \Gamma_{\mathrm{c}}=1000, R_{\mathrm{c}} / c=1\right)$. Other parameters and the symbols are the same as those adopted in Fig. 12.

\section{Discussion and conclusions}

In this paper, we investigate how the Doppler effect in the fireball framework affects the observed spectrum of the object with various rest frame emission or absorption lines.

In Sect. 2, we study the effect on narrow emission lines. The study reveals that, influenced by the Doppler effect in the fireball framework, rest frame narrow emission lines would significantly shift to higher energy bands and would be significantly broadened. A rest frame narrow emission line at high energy bands could form an up-turning tip of the high energy tail in the observed spectrum. It shows that, when $\Gamma$ is large enough, the observed line frequency $v_{\text {line }}$ and the rest frame line frequency $v_{0, \text { line }}$ would be related by $v_{\text {line }} \approx 2 \Gamma v_{0, \text { line }}$. It also 


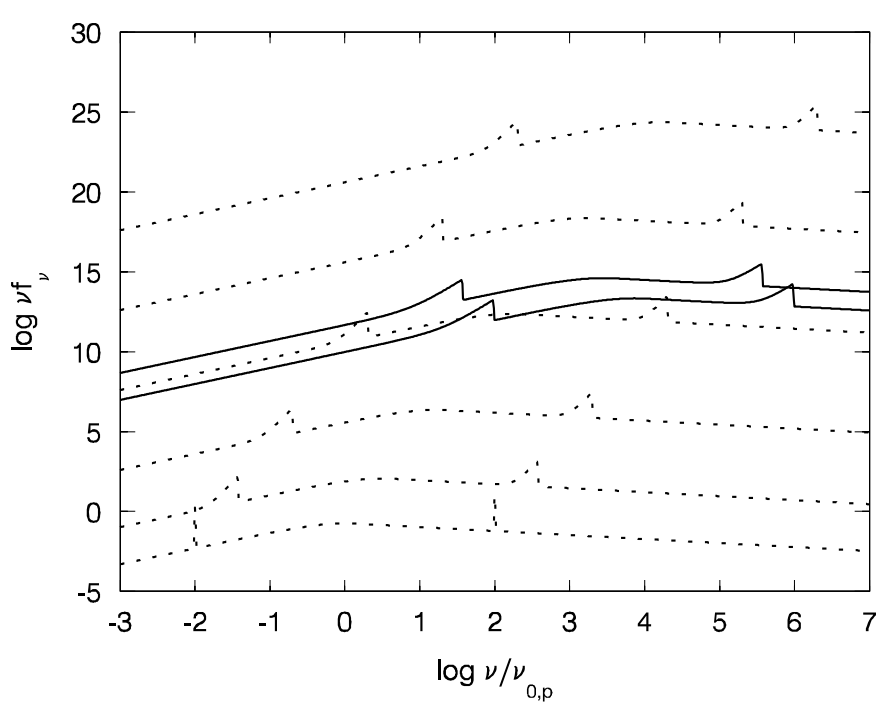

Fig. 16. The expected spectrum of a fireball with a variable Lorentz factor, where $\Gamma_{\mathrm{c}}=10000$ and $k=10 k_{0}\left(p=10, \Gamma_{\mathrm{c}}=10000, R_{\mathrm{c}} / c=\right.$ 1). Other parameters and the symbols are the same as those adopted in Fig. 12.

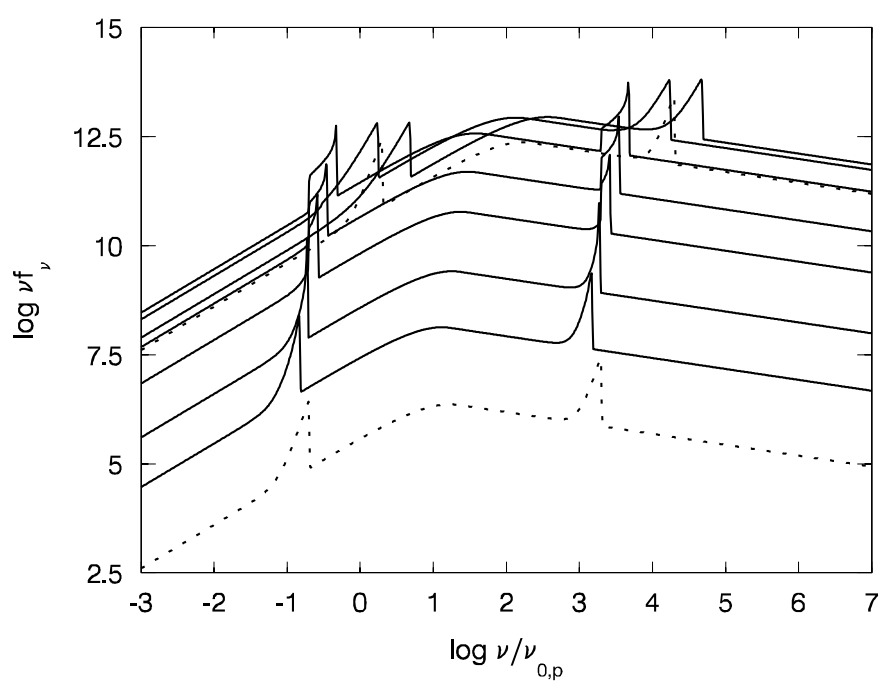

Fig. 17. The expected spectrum of a fireball with a variable Lorentz factor, where we take $p=10$ and $k=10 k_{0}\left(p=10, \Gamma_{\mathrm{c}}, R_{\mathrm{c}} / c=\right.$ 1) and consider different values of the initial Lorentz factor $\Gamma_{c}$. The solid lines from the bottom to the top correspond to $\Gamma_{\mathrm{c}}=$ $10,20,50,100,200,500,1000$, respectively, and the dotted lines from the bottom to the top are the $\Gamma=10$ and $\Gamma=100$ lines presented in Fig. 1. Other parameters are the same as those adopted in Fig. 12.

reveals that the relative width of the line feature would approach an asymptotic value when $\Gamma \gg 1$. In the case where the relative width of the rest frame narrow emission lines is 0.0235 , the observed $\Delta v_{F W H M} / v_{\text {line }}$ would become almost one magnitude larger than that of the rest frame emission line. When the rest frame line is narrow enough, the relative width would approach 0.162 .

In Sect. 3, the effects on other lines are investigated. The study shows that in the spectrum of a relativistically expanding fireball, any rest frame lines would shift to higher energy bands and would be significantly smoothed. Rest frame weak

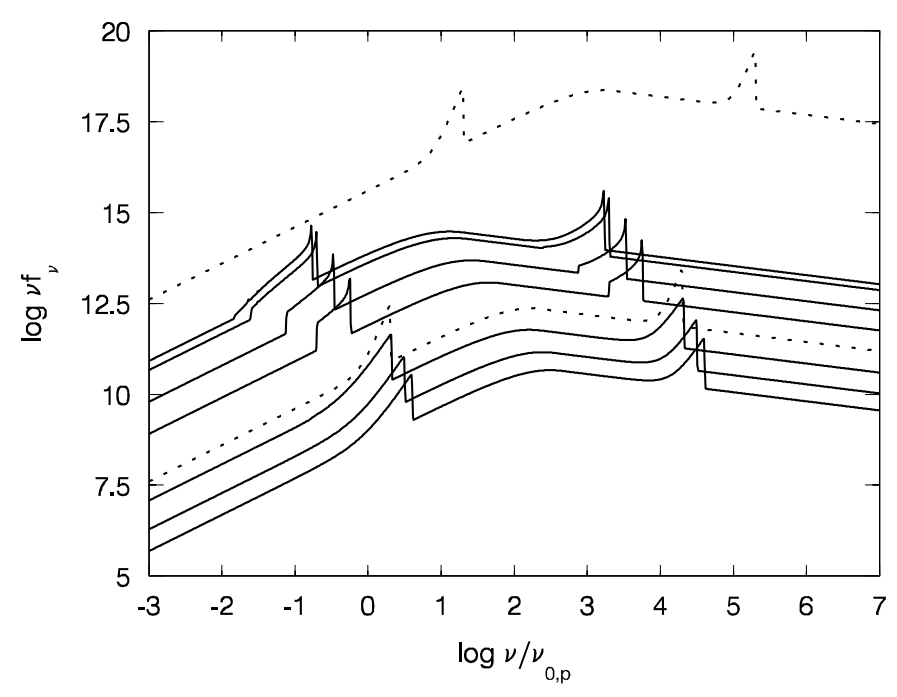

Fig. 18. The expected spectrum of a fireball with a variable Lorentz factor, where we take $\Gamma_{\mathrm{c}}=300$ and $k=10 k_{0}\left(p=10, \Gamma_{\mathrm{c}}=300, R_{\mathrm{c}} / c=\right.$ 1) and consider different observation times, which are represented by different values of $p$, respectively. The solid lines from the bottom to the top correspond to $p=0,1,3,10,100,1000,2000$, respectively, and the dotted lines from the bottom to the top are the $\Gamma=100$ and $\Gamma=1000$ lines presented in Fig. 1. Other parameters are the same as those adopted in Fig. 12.

narrow emission lines as well as narrow absorption lines and absorption line forests would be smoothed and would hardly be detectable. Meanwhile, the features of rest frame broad emission lines as well as both strong and weak broad absorption lines would remain almost unchanged and therefore would be easier to detect. Deep gaps caused by rest frame broad absorption lines would be significantly filled. In addition, each of the rest frame emission line forests would form a single broad line feature.

In Sect. 4, we investigate the effect of time dependence of the line intensity and the effect of variation of the Lorentz factor. The study reveals that, in the spectrum of a relativistically expanding fireball, rest frame dimming narrow emission lines would also shift to higher energy bands and would be significantly broadened. However, when the Lorentz factor is very large (say, $\Gamma>1000$ ), the line feature would disappear. As time progresses, the feature would eventually disappear and this would occur at a much earlier time for those fireballs with a larger value of $\Gamma$. Considering the variation of the Lorentz factor of fireballs we find that the flux observed at a relatively later time would be larger than that observed at an earlier time, suggesting that, in the situation studied here, the enhancement of the radius of the fireball plays an important role in determining the magnitude of the flux. When the initial Lorentz factor is very small, the effect of deceleration would be insignificant, and the positions of emission lines would remain almost unchanged. When the initial Lorentz factor is large enough, due to the deceleration, the flux would become much smaller than the constant radiation flux and the change of the positions of emission lines would be obvious. As time progresses, the positions would shift to lower energy bands. In addition, we find an unfamiliar form of emission lines that is sharp on both edges. 
Further study reveals that this phenomenon depends not only on the initial Lorentz factor but also on the observation time. For some initial Lorentz factors, the shape of lines changes with time. This might become an interesting observational characteristic expected in GRBs.

One can conclude that, for a relativistically expanding fireball, there would be no narrow emission lines expected in its spectrum, and rest frame narrow absorption lines as well as rest frame emission or absorption line forests would not be detectable. Since $v_{\text {line }}$ is a linear function of $\Gamma$, features of lines could serve as an indicator of the expansion speed of fireballs as long as they are identified. In the phase of deceleration of the Lorentz factor, one can expect a change of the shape of emission lines of fireballs with time.

If there are some high energy emission lines such as the $6.4 \mathrm{keV}$ line and the $511 \mathrm{keV}$ annihilation line in the outer shell of fireballs, we could expect them to appear at around $0.1 \mathrm{MeV}$ and $10 \mathrm{MeV}$ respectively when $\Gamma \sim 10$, or $1 \mathrm{MeV}$ and $100 \mathrm{MeV}$ when $\Gamma \sim 100$, or $10 \mathrm{MeV}$ and $1000 \mathrm{MeV}$ when $\Gamma \sim 1000$. Regarding the previous report of the detection of emission features near $400 \mathrm{keV}$ (Mazets et al. 1980), we would prefer to interpret them as the blue-shifted $6.4 \mathrm{keV}$ line corresponding to the fireball expanding with $\Gamma \sim 30$. Due to the great blue shift effect, the conventional redshift interpretation of the line seems unlikely. We propose that, in detecting line features, the blue shift and broadening effects due to the expansion of the fireball should be taken into account.

In addition to the conventional fireball model, the method can also be applied to the cannonball model (Dado et al. 2002a, 2002b). To explain the X-ray line features observed in some GRBs as being Doppler-boosted, Dado et al. (2003) employed a cannonball model for which the radius of the cannonball was treated as a constant and the direction of the motion of the cannonball was assumed to be unchanged. In this situation, some formulas in Paper I can be simply applied. Taking $t_{0, \theta}-t_{0, \mathrm{c}}$ as the proper emission time $t_{0}$ and $t-t_{\mathrm{c}}-D / c+\left(R_{\mathrm{c}} / c\right) \cos \theta$ as the observation time $t$ we would get from (A.8) of Paper I that $t_{0}=\delta(t) t$, where $\delta(t) \equiv 1 / \Gamma(t)(1-\beta(t) \cos \theta)$ is the Doppler factor of the cannonball at time $t$. The observed area of the cannonball is $S(\theta, \Gamma) R^{2}$, where $S(\theta, \Gamma)$ is a function of the Lorentz factor, which would be $\pi$ when the contraction effect due to the relative motion is ignored. Replacing $R^{2}\left(t_{\theta}\right) \cos \theta \sin \theta \mathrm{d} \theta \mathrm{d} \varphi$ with $S(\theta, \Gamma) R^{2}$ we get from (A.11) of Paper I that $\mathrm{d} E=$ $\Delta s_{\mathrm{ob}} \mathrm{d} v \mathrm{~d} t S(\theta, \Gamma) R^{2} I_{\nu}(v, t) / D^{2}$, where the cosmological effect is ignored. Absorbing $S(\theta, \Gamma)$ into the normalized coefficient we get $F_{\text {obs }}(v, t)=R^{2} I_{v}(v, t) / D^{2}$. Applying the Doppler effect and replacing $I_{v}(v, t)$ with $\delta^{3}(t) I_{0, v}\left(v_{0}, t_{0}\right)=\delta^{3}(t) F_{\mathrm{CB}}[v / \delta(t), \delta(t) t]$ we come to Eq. (3) of Dado et al. (2003) and the Doppler effect itself leads to Eq. (6) there (when the cosmological effect is ignored); based on this the spectral data (including the X-ray line features) of some GRBs were fitted (Dado et al. 2003).

Acknowledgements. It is my great pleasure to thank Prof. M. J. Rees for his helpful suggestions and discussion. This work was supported by the Special Funds for Major State Basic Research Projects (“973”) and National Natural Science Foundation of China (No. 10273019).

\section{References}

Band, D., Matteson, J., Ford, L., et al. 1993, ApJ, 413, 281

Band, D. L., Ryder, S., Ford, L. A., et al. 1996, ApJ, 458, 746

Dado, S., Dar, A., \& De Rujula, A. 2002a, A\&A, 388, 1079

Dado, S., Dar, A., \& De Rujula, A. 2002b, ApJ, 572, L143

Dado, S., Dar, A., \& De Rujula, A. 2003, ApJ, 585, 890

Fenimore, E. E., Conner, J. P., Epstein, R. I., et al. 1988, ApJ, 335, L71

Ford, L. A., Band, D. L., Matteson, J. L., et al. 1995, ApJ, 439, 307

Goodman, J. 1986, ApJ, 308, L47

Katz, J. I. 1994, ApJ, 422, 248

Krolik, J. H., \& Pier, E. A. 1991, ApJ, 373, 277

Liang, E. P., Ternigan, T. E., \& Rodrigues, R. 1983, ApJ, 271, 766

Mazets, E. P., Golenetskii, S. V., Aptekar, R. L., Guryan, Y. A., \& Ilinskii, V. N. 1980, Sov. Astron. Lett., 6, 372

Meszaros, P., \& Rees, M. J. 1993, ApJ, 405, 278

Meszaros, P., \& Rees, M. J. 1994, MNRAS, 269, L41

Meszaros, P., \& Rees, M. J. 1998, ApJ, 502, L105

Metzger, M. R., Djorgovski, S. G., Kulkarni, S. R., et al. 1997, Nature, 387,878

Murakami, T., Fujii, M., Hayashida, K., Itoh, M., \& Nishimura, J. 1988, Nature, 335, 234

Paczynski, B. 1986, ApJ, 308, L43

Paczynski, B., \& Xu, G. 1994, ApJ, 427, 708

Palmer, D. M., Teegarden, B. J., Schaefer, B. E., et al. 1994, ApJ, 433, L77

Piran, T. 1999, Phys. Rept., 314, 575

Preece, R. D., Pendleton, G. N., Briggs, M. S., et al. 1998, ApJ, 496, 849

Preece, R. D., Briggs, M. S., Mallozzi, R. S., et al. 2000, ApJS, 126, 19

Ramaty, R., \& Meszaros, P. 1981, ApJ, 250, 384

Qin, Y.-P. 2002, A\&A, 396, 705 (Paper I)

Rees, M. J., \& Meszaros, P. 1992, MNRAS, 258, 41p

Rees, M. J., \& Meszaros, P. 1994, ApJ, 430, L93

Sari, R., Narayan, R., \& Piran, T. 1996, ApJ, 473, 204

Schaefer, B. E., Teegarden, B. J., Fantasia, S. F., et al. 1994, ApJS, 92, 285 


\section{Online Material}




\section{Appendix A: Analytical study of $\Delta v_{F W H M} / v_{\text {line }}$ for very narrow emission lines}

Here we calculate $\Delta v_{F W H M} / v_{\text {line }}$ analytically for very narrow emission lines when $\Gamma \gg 1$.

The flux expected from a fireball with a definite value of the Lorentz factor is (see Paper I)

$f_{v}(t)=\frac{2 \pi \widetilde{R}^{2}(t)}{D^{2} \Gamma^{3}} \int_{\theta_{\min }}^{\theta_{\max }} \frac{I_{0, v}\left(t_{0, \theta}, v_{0, \theta}\right) \cos \theta \sin \theta}{(1-\beta \cos \theta)^{5}} \mathrm{~d} \theta$,

where $\widetilde{R}(t)$ is determined by (2); $t, v, D, \theta, v_{0, \theta}, t_{0, \theta}$, and $I_{0, v}\left(t_{0, \theta}, v_{0, \theta}\right)$ are explained in Sect. 2. As discussed in Sect. 2, we consider a constant radiation and get the following by applying (3):

$f_{v}(t)=\frac{2 \pi I_{0} \widetilde{R}^{2}(t)}{D^{2} \Gamma^{3}} \int_{\theta_{\min }}^{\theta_{\max }} g_{0, v}\left(v_{0, \theta}\right) \frac{\cos \theta \sin \theta}{(1-\beta \cos \theta)^{5}} \mathrm{~d} \theta$.

To make an analytical study of $\Delta v_{F W H M} / v_{\text {line }}$ for very narrow emission lines, one would prefer a simple form of emission, which can be calculated analytically and can represent an emission line in extreme cases.

Let us consider a very narrow rectangle of emission. The rest frame radiation is assumed to be

$g_{0, v}\left(v_{0, \theta}\right)=g_{0, v, \mathrm{c}}\left(v_{0, \theta}\right)+e_{0, v}\left(v_{0, \theta}\right)$

where, while $g_{0, v, \mathrm{c}}\left(v_{0, \theta}\right)$ describes the relative intensity of a continuum (see Sect. 2), $e_{0, v}\left(v_{0, \theta}\right)$ stands for the relative intensity of an emission line which is

$e_{0, v}\left(v_{0, \theta}\right)=h \quad\left(v_{0, \text { line }} \leq v_{0, \theta} \leq v_{0, \text { line }}+\Delta v_{0, \text { line }}\right)$

where $h$ is a constant.

To calculate $\Delta v_{F W H M} / v_{\text {line }}$, one needs only to deal with the radiation of the line:

$f_{v, \text { line }}(t)=\frac{2 \pi I_{0} \widetilde{R}^{2}(t)}{D^{2} \Gamma^{3}} e_{v}$

with

$e_{v} \equiv h \int_{\theta_{\min }}^{\theta_{\max }} \frac{\cos \theta \sin \theta}{(1-\beta \cos \theta)^{5}} \mathrm{~d} \theta$

According to the Doppler effect, for certain values of $v_{0, \theta}$ and $\Gamma$, the shifted frequency $v$ is confined in the range $v_{0, \theta} / \Gamma \leq v \leq$ $v_{0, \theta} / \Gamma(1-\beta)$ for an expanding fireball. Thus, the valid range of frequency in (A.6) is

$\frac{v_{0, \text { line }}}{\Gamma} \leq v \leq \frac{v_{0, \text { line }}+\Delta v_{0, \text { line }}}{\Gamma(1-\beta)}$.

The integral limits $\theta_{\min }$ and $\theta_{\max }$ in (A.6) can be determined by (Paper I)

$$
\begin{gathered}
\theta_{\min }=\cos ^{-1}\left(\min \left\{1, \frac{1}{\beta}\left(1-\frac{v_{0, \text { line }}}{\Gamma v}\right)\right\}\right) \\
\left(0 \leq \frac{1}{\beta}\left(1-\frac{v_{0, \text { line }}}{\Gamma v}\right) ; 0<\beta\right)
\end{gathered}
$$

and

$$
\begin{gathered}
\theta_{\max }=\cos ^{-1}\left(\max \left\{0, \frac{1}{\beta}\left(1-\frac{v_{0, \text { line }}+\Delta v_{0, \text { line }}}{\Gamma v}\right)\right\}\right) \\
\left(\frac{1}{\beta}\left(1-\frac{v_{0, \text { line }}+\Delta v_{0, \text { line }}}{\Gamma v}\right) \leq 1 ; 0<\beta\right) .
\end{gathered}
$$

Integrating (A.6) we get

$$
\begin{aligned}
e_{v}= & \frac{h}{\beta^{2}}\left\{\frac { 1 } { 4 } \left[\frac{1}{\left(1-\beta \min \left\{1, \frac{1}{\beta}\left(1-\frac{v_{0, \text { line }}}{\Gamma v}\right)\right\}\right)^{4}}\right.\right. \\
& \left.-\frac{1}{\left(1-\beta \max \left\{0, \frac{1}{\beta}\left(1-\frac{v_{0, \text { line }}+\Delta v_{0, \text { line }}}{\Gamma v}\right)\right\}\right)^{4}}\right] \\
& +\frac{1}{3}\left[\frac{1}{\left(1-\beta \max \left\{0, \frac{1}{\beta}\left(1-\frac{v_{0, \text { line }}+\Delta v_{0, \text { line }}}{\Gamma v}\right)\right\}\right)^{3}}\right. \\
& \left.\left.-\frac{1}{\left(1-\beta \min \left\{1, \frac{1}{\beta}\left(1-\frac{v_{0, \text { line }}}{\Gamma v}\right)\right\}\right)^{3}}\right]\right\} \\
& \left(\frac{v_{0, \text { line }}}{\Gamma} \leq v \leq \frac{v_{0, \text { line }}+\Delta v_{0, \text { line }}}{\Gamma(1-\beta)}\right) .
\end{aligned}
$$

Let us calculate $\partial e_{v} / \partial v$ in different frequency ranges: range I, $\left(\frac{v_{0, \text { line }}}{\Gamma} \leq v \leq \frac{v_{0, \text { line }}+\Delta v_{0, \text { line }}}{\Gamma}\right)$; range II, $\left(\frac{v_{0, \text { line }}+\Delta v_{0, \text { line }}}{\Gamma} \leq v \leq \frac{v_{0, \text { line }}}{\Gamma(1-\beta)}\right)$; and range III, $\left(\frac{v_{0, \text { line }}}{\Gamma(1-\beta)} \leq v \leq \frac{v_{0, \text { line }}+\Delta v_{0, \text { line }}}{\Gamma(1-\beta)}\right)$. Here, we assume

$$
\frac{\Delta v_{0, \text { line }}}{v_{0, \text { line }}} \ll 1 \quad \text { and } \quad 1 \ll \Gamma,
$$

which leads to

$v_{0, \text { line }}+\Delta v_{0, \text { line }} \ll \frac{v_{0, \text { line }}}{1-\beta}$.

Applying (A.12), we find $\partial e_{v} / \partial v \geq 0$ in both ranges I and II, and $\partial e_{v} / \partial v<0$ in range III. This leads to

$$
\begin{aligned}
e_{v, \text { max }}= & \frac{h}{3 \beta^{2}(1-\beta)^{3}}\left\{\frac{3\left[1-\frac{v_{0, \text { line }}^{4}}{\left(v_{0, \text { line }}+\Delta v_{0, \text { line }}\right)^{4}}\right]}{4(1-\beta)}\right. \\
& \left.+\frac{v_{0, \text { line }}^{3}}{\left(v_{0, \text { line }}+\Delta v_{0, \text { line }}\right)^{3}}-1\right\},
\end{aligned}
$$

which is the maximum value of $e_{v}$ in the whole frequency range.

The frequency at which $e_{v, \max }$ is found is identified as the observed line frequency $v_{\text {line }}$ which is determined by

$v_{\text {line }}=\frac{v_{0, \text { line }}}{\Gamma(1-\beta)}$.

When $\Gamma \gg 1$, it would approach $v_{\text {line }} \simeq 2 \Gamma v_{0, \text { line }}$.

With (A.13), we first calculate $v_{F W H M}$ in region II, $v_{F W H M, I I}$, and obtain 


$$
\begin{aligned}
& \frac{\Gamma^{4} v_{F W H M, \text { II }}^{4}}{4 v_{0, \text { line }}^{4}} {\left[1-\frac{v_{0, \text { line }}^{4}}{\left(v_{0, \text { line }}+\Delta v_{0, \text { line }}\right)^{4}}\right]-\frac{1}{8(1-\beta)^{4}} } \\
& \times {\left[1-\frac{v_{0, \text { line }}^{4}}{\left(v_{0, \text { line }}+\Delta v_{0, \text { line }}\right)^{4}}\right]=\frac{\Gamma^{3} v_{F W H M, \text { II }}^{3}}{3 v_{0, \text { line }}^{3}} } \\
& \times\left[1-\frac{v_{0, \text { line }}^{3}}{\left(v_{0, \text { line }}+\Delta v_{0, \text { line }}\right)^{3}}\right] \\
&-\frac{1}{6(1-\beta)^{3}}\left[1-\frac{v_{0, \text { line }}^{3}}{\left(v_{0, \text { line }}+\Delta v_{0, \text { line }}\right)^{3}}\right] .
\end{aligned}
$$

The solution of (A.15) can be approximated by

$$
\begin{aligned}
\frac{\Gamma v_{F W H M, \mathrm{II}}}{v_{0, \text { line }}} \simeq & \frac{\left[1+3\left(2^{1 / 12}-1\right)\right]^{1 / 3}}{2^{1 / 3}(1-\beta)} \\
& \times\left[1+\frac{2\left(2^{1 / 12}-1\right)}{1+3\left(2^{1 / 12}-1\right)} \frac{\Delta v_{0, \text { line }}}{v_{0, \text { line }}}\right] .
\end{aligned}
$$

Second, we calculate $v_{F W H M}$ in region III, $v_{F W H M, I I}$, and get

$$
\begin{aligned}
\frac{\Gamma^{4} v_{F W H M, \text { III }}^{4}}{4 v_{0, \text { line }}^{4}}- & \frac{1+\left(1+\frac{\Delta v_{0, \text { line }}}{v_{0, \text { line }}}\right)^{4}}{8(1-\beta)^{4}} \\
& =\frac{\Gamma^{3} v_{F W H M, \text { III }}^{3}\left(1+\frac{\Delta v_{0, \text { line }}}{v_{0, \text { line }}}\right)}{3 v_{0, \text { line }}^{3}} \\
- & \frac{\left(1+\frac{\Delta v_{0, \text { line }}}{v_{0, \text { line }}}\right)\left[1+\left(1+\frac{\Delta v_{0, \text { line }}}{v_{0, \text { line }}}\right)^{3}\right]}{6(1-\beta)^{3}} .
\end{aligned}
$$

The solution of (A.17) can be approximated by

$$
\begin{aligned}
\frac{\Gamma v_{F W H M, \text { III }}}{v_{0, \text { line }}} & \simeq \frac{1}{2^{1 / 3}(1-\beta)}\left(2^{1 / 3}+2^{-2 / 3} \frac{\Delta v_{0, \text { line }}}{v_{0, \text { line }}}\right) \\
& =\frac{1+\frac{1}{2} \frac{\Delta v_{0, \text { line }}}{v_{0, \text { line }}}}{1-\beta} .
\end{aligned}
$$

We therefore obtain

$$
\begin{aligned}
\frac{\Delta v_{F W H M}}{v_{\text {line }}} \simeq & 1-\left[\frac{1+3\left(2^{1 / 12}-1\right)}{2}\right]^{1 / 3} \\
& +\left\{\frac{1}{2}-\frac{2^{2 / 3}\left(2^{1 / 12}-1\right)}{\left[1+3\left(2^{1 / 12}-1\right)\right]^{2 / 3}}\right\} \frac{\Delta v_{0, \text { line }}}{v_{0, \text { line }}} \\
\simeq & 0.162+0.415 \frac{\Delta v_{0, \text { line }}}{v_{0, \text { line }}} .
\end{aligned}
$$

When $\Delta v_{0, \text { line }} / v_{0, \text { line }} \rightarrow 0$, one will get $\Delta v_{F W H M} / v_{\text {line }} \simeq 0.162$

\section{Appendix B: Expected flux of a fireball with a variable Lorentz factor}

We examine a fireball expanding at a variable velocity $v=\beta c$ and employ the same coordinate system adopted in Sect. 2. Consider radiation from a rest frame differential surface, $\mathrm{d} s_{0, \theta, \varphi}$, of the fireball at proper time $t_{0, \theta}$. Let $\mathrm{d} s_{\theta, \varphi}$ be the corresponding differential surface resting on the observer's framework, coinciding with $\mathrm{d} s_{0, \theta, \varphi}$ at $t_{0, \theta}$, and let $t_{\theta}$ be the corresponding coordinate time of $t_{0, \theta}$. Obviously, $t_{\theta}=t_{\theta}\left(t_{0, \theta}\right)$.

According to the theory of special relativity, $t_{\theta}$ and $t_{0, \theta}$ are related by

$\mathrm{d} t_{\theta}=\Gamma\left(t_{\theta}\right) \mathrm{d} t_{0, \theta}=\Gamma_{0}\left(t_{0, \theta}\right) \mathrm{d} t_{0, \theta}$,

where $\Gamma\left(t_{\theta}\right)$ is the Lorentz factor of the fireball at $t_{\theta}$ and $\Gamma_{0}\left(t_{0, \theta}\right) \equiv \Gamma\left[t_{\theta}\left(t_{0, \theta}\right)\right]$. Integrating (B.1) yields

$t_{\theta}=\int_{t_{0, \mathrm{c}}}^{t_{0, \theta}} \Gamma_{0}\left(t_{0, \theta}\right) \mathrm{d} t_{0, \theta}+t_{\mathrm{c}}$ or $t_{0, \theta}=\int_{t_{\mathrm{c}}}^{t_{\theta}} \frac{1}{\Gamma\left(t_{\theta}\right)} \mathrm{d} t_{\theta}+t_{0, \mathrm{c}}$

here we assign $t_{\theta}=t_{\mathrm{c}}$ when $t_{0, \theta}=t_{0, \mathrm{c}}$.

The area of $\mathrm{d} s_{\theta, \varphi}$ is

$\mathrm{d} s_{\theta, \varphi}=R^{2}\left(t_{\theta}\right) \sin \theta \mathrm{d} \theta \mathrm{d} \varphi$,

where $R\left(t_{\theta}\right)$ is the radius of the fireball at $t_{\theta}$. Suppose the radius develops as

$\mathrm{d} R\left(t_{\theta}\right)=c \beta\left(t_{\theta}\right) \mathrm{d} t_{\theta}$.

Integrating (B.4) leads to

$R\left(t_{\theta}\right)=c \int_{t_{\mathrm{c}}}^{t_{\theta}} \beta\left(t_{\theta}\right) \mathrm{d} t_{\theta}+R_{\mathrm{c}}$,

where $R_{\mathrm{c}}$ is the radius at time $t_{\theta}=t_{\mathrm{c}}$. As assigned above, $t_{\theta}$ and $t_{0, \theta}$ correspond to the same moment. Therefore the radius can be expressed as

$$
\begin{aligned}
R_{0}\left(t_{0, \theta}\right) & \equiv R\left[t_{\theta}\left(t_{0, \theta}\right)\right]=c \int_{t_{\mathrm{c}}}^{t_{\theta}\left(t_{0, \theta}\right)} \beta\left(t_{\theta}\right) \mathrm{d} t_{\theta}+R_{\mathrm{c}} \\
& =c \int_{t_{0, \mathrm{c}}}^{t_{0, \theta}} \beta_{0}\left(t_{0, \theta}\right) \Gamma_{0}\left(t_{0, \theta}\right) \mathrm{d} t_{0, \theta}+R_{\mathrm{c}},
\end{aligned}
$$

where (B.1) is applied, and $\beta_{0}\left(t_{0, \theta}\right) \equiv \beta\left[t_{\theta}\left(t_{0, \theta}\right)\right]$.

Let us consider an observation within small intervals $t-$ $t+\mathrm{d} t$ and $v-v+\mathrm{d} v$ carried out by an observer with a detector $\triangle s_{\text {ob }}$ at a distance $D(D$ is the distance between the observer and the center of the fireball), where $D \gg R\left(t_{\theta}\right)$. Suppose radiation from $\mathrm{d} s_{0, \theta, \varphi}$ reaching the observer within the above observation intervals is emitted within the proper time interval $t_{0, \theta}-t_{0, \theta}+$ $\mathrm{d} t_{0, \theta}$ and the rest frame frequency interval $v_{0, \theta}-v_{0, \theta}+\mathrm{d} v_{0, \theta}$. According to the Doppler effect, $v$ and $v_{0, \theta}$ are related by

$v=\frac{v_{0, \theta}}{\Gamma\left(t_{\theta}\right)\left[1-\beta\left(t_{\theta}\right) \cos \theta\right]}=\frac{v_{0, \theta}}{\Gamma_{0}\left(t_{0, \theta}\right)\left[1-\beta_{0}\left(t_{0, \theta}\right) \cos \theta\right]}$.

Considering the travelling of light from the fireball to the observer we get

$c\left(t-t_{\theta}\right)=D-R\left(t_{\theta}\right) \cos \theta$

(here the cosmological effect is ignored). Combining (B.5) and (B.8) we get

$\left[\int_{t_{\mathrm{c}}}^{t_{\theta}} \beta\left(t_{\theta}\right) \mathrm{d} t_{\theta}\right] \cos \theta-t_{\theta}=D / c-t-\left(R_{\mathrm{c}} / c\right) \cos \theta$,

while combining (B.2), (B.6) and (B.8) we obtain

$$
\begin{array}{r}
{\left[\int_{t_{0, \mathrm{c}}}^{t_{0, \theta}} \beta_{0}\left(t_{0, \theta}\right) \Gamma_{0}\left(t_{0, \theta}\right) \mathrm{d} t_{0, \theta}\right] \cos \theta-\left[\int_{t_{0, \mathrm{c}}}^{t_{0, \theta}} \Gamma_{0}\left(t_{0, \theta}\right) \mathrm{d} t_{0, \theta}\right]} \\
=D / c-t-\left(R_{\mathrm{c}} / c\right) \cos \theta+t_{\mathrm{c}} .
\end{array}
$$


Once $\beta\left(t_{\theta}\right)$ or $\beta_{0}\left(t_{0, \theta}\right)$ is known, $t_{\theta}$ and $t_{0, \theta}$ as functions of $\theta$ and $t$ can be determined by (B.9) and (B.10), respectively. Thus the radius of the fireball can be determined by (B.5) or (B.6).

Suppose photons, which are emitted from $\mathrm{d} s_{0, \theta, \varphi}$ within the proper time interval $t_{0, \theta}-t_{0, \theta}+\mathrm{d} t_{0, \theta}$ and then reach the observer within $t-t+\mathrm{d} t$, pass through $\mathrm{d} s_{\theta, \varphi}$ within the coordinate time interval $t_{\theta}-t_{\theta}+\mathrm{d} t_{\theta, s}$. Since both $\mathrm{d} s_{\theta, \varphi}$ and the observer rest in the same framework, $\mathrm{d} t_{\theta, s}=\mathrm{d} t$. Of course, the frequency interval for the photons measured by both $\mathrm{d} s_{\theta, \varphi}$ and the observer must be the same, which is taken as $v-v+\mathrm{d} v$. In views of $\mathrm{d} s_{\theta, \varphi}$, the amount of energy emitted from $\mathrm{d} s_{0, \theta, \varphi}$ within $t_{0, \theta}-t_{0, \theta}+\mathrm{d} t_{0, \theta}$ and $v_{0, \theta}-v_{0, \theta}+\mathrm{d} v_{0, \theta}$ towards the observer would be

$\mathrm{d} E_{\theta, \varphi}=I_{\nu}\left(t_{\theta}, v, \theta\right) \cos \theta \mathrm{d} s_{\theta, \varphi} \mathrm{d} \omega \mathrm{d} \nu \mathrm{d} t$,

where $I_{v}\left(t_{\theta}, v, \theta\right)$ is the intensity of radiation measured by $\mathrm{d} s_{\theta, \varphi}$, which is allowed to be a function of direction, and $\mathrm{d} \omega$ is the solid angle of $\triangle s_{\mathrm{ob}}$ with respect to the fireball, which is

$\mathrm{d} \omega=\frac{\triangle s_{\mathrm{ob}}}{D^{2}}$.

Thus,

$\mathrm{d} E_{\theta, \varphi}=\frac{\Delta s_{\mathrm{ob}} \mathrm{d} \nu \mathrm{d} t R^{2}\left(t_{\theta}\right) I_{\nu}\left(t_{\theta}, v, \theta\right) \cos \theta \sin \theta \mathrm{d} \theta \mathrm{d} \varphi}{D^{2}}$,

where (B.3) and (B.12) are applied.

The total amount of energy emitted from the whole fireball surface detected by the observer within the above observation intervals is an integral of $\mathrm{d} E_{\theta, \varphi}$ over that area, which is

$\mathrm{d} E=\frac{2 \pi \Delta s_{\mathrm{ob}} \mathrm{d} \nu \mathrm{d} t}{D^{2}} \int_{\theta_{\min }}^{\theta_{\max }} R^{2}\left(t_{\theta}\right) I_{v}\left(t_{\theta}, v, \theta\right) \cos \theta \sin \theta \mathrm{d} \theta$,

where $\theta_{\min }$ and $\theta_{\max }$ are determined by the fireball surface itself together with the emitted ranges of $t_{0, \theta}$ and $v_{0, \theta}$. Thus, the expected flux would be

$f_{v}(t)=\frac{2 \pi}{D^{2}} \int_{\theta_{\min }}^{\theta_{\max }} R^{2}\left(t_{\theta}\right) I_{v}\left(t_{\theta}, v, \theta\right) \cos \theta \sin \theta \mathrm{d} \theta$.

It is well known that the observer frame intensity $I_{v}\left(t_{\theta}, v, \theta\right)$ is related to the rest frame intensity $I_{0, v}\left(t_{0, \theta}, v_{0, \theta}, \theta\right)$ by

$I_{\nu}\left(t_{\theta}, v, \theta\right)=\left(\frac{v}{v_{0, \theta}}\right)^{3} I_{0, v}\left(t_{0, \theta}, v_{0, \theta}, \theta\right)$.

The flux then can be written as

$f_{v}(t)=\frac{2 \pi}{D^{2}} \int_{\theta_{\min }}^{\theta_{\max }} \frac{R_{0}^{2}\left(t_{0, \theta}\right) I_{0, v}\left(t_{0, \theta}, v_{0, \theta}, \theta\right) \cos \theta \sin \theta}{\Gamma_{0}^{3}\left(t_{0, \theta}\right)\left[1-\beta_{0}\left(t_{0, \theta}\right) \cos \theta\right]^{3}} \mathrm{~d} \theta$,

where (B.6) and (B.7) are applied.

The range of $\theta$ of the visible fireball surface is

$0 \leq \theta \leq \pi / 2$.

Within this range, suppose the emitted ranges of $t_{0, \theta}$ and $v_{0, \theta}$ constrain $\theta$ by

$\theta_{t, \min } \leq \theta \leq \theta_{t, \max }$

and

$\theta_{v, \min } \leq \theta \leq \theta_{v, \max }$, respectively. Then when the following condition

$\max \left\{\theta_{t, \min }, \theta_{v, \min }\right\}<\min \left\{\theta_{t, \max }, \theta_{v, \max }\right\}$

is satisfied, $\theta_{\min }$ and $\theta_{\max }$ would be obtained by

$\theta_{\min }=\max \left\{\theta_{t, \min }, \theta_{v, \min }\right\}$

and

$\theta_{\max }=\min \left\{\theta_{t, \max }, \theta_{v, \max }\right\}$

respectively.

Let the emitted ranges of $t_{0, \theta}$ and $v_{0, \theta}$ be

$t_{0, \min } \leq t_{0, \theta} \leq t_{0, \max }$

and

$v_{0, \min } \leq v_{0, \theta} \leq v_{0, \max }$,

respectively. For $\beta>0$, one can obtain the following from (B.7) and (B.25):

$$
\begin{aligned}
\theta_{v, \text { min }}\left(t_{0, \theta}\right)= & \cos ^{-1}\left(\min \left\{1, \frac{1}{\beta}\left(1-\frac{v_{0, \min }}{\Gamma v}\right)\right\}\right) \\
& \left(0 \leq \frac{1}{\beta}\left(1-\frac{v_{0, \min }}{\Gamma v}\right) ; 0<\beta\right)
\end{aligned}
$$

and

$$
\begin{aligned}
\theta_{\nu, \text { max }}\left(t_{0, \theta}\right)= & \cos ^{-1}\left(\max \left\{0, \frac{1}{\beta}\left(1-\frac{v_{0, \text { max }}}{\Gamma v}\right)\right\}\right) \\
& \left(\frac{1}{\beta}\left(1-\frac{v_{0, \max }}{\Gamma \nu}\right) \leq 1 ; 0<\beta\right),
\end{aligned}
$$

where $\beta=\beta\left(t_{\theta}\right)=\beta_{0}\left(t_{0, \theta}\right)$ and $\Gamma=\Gamma\left(t_{\theta}\right)=\Gamma_{0}\left(t_{0, \theta}\right)$. From (B.10) and (B.24) one can also determine $\theta_{t, \min }$ and $\theta_{t, \max }$ once $\beta\left(t_{\theta}\right)$ or $\beta_{0}\left(t_{0, \theta}\right)$ is known.

\section{Appendix C: Lower limit of the coefficient of deceleration}

We consider in Sect. 4 the case of the radiative hydrodynamics for which, when decelerated, the development of the Lorentz factor of a fireball follows that shown by (44). The coordinate time, $t_{\theta}$, of the differential surface of the fireball concerned and the observation time, $t$, are related by (B.9). Since $\beta\left(t_{\theta}\right) \leq \beta_{\mathrm{c}}$, where $\beta_{\mathrm{c}} \equiv \sqrt{\Gamma_{\mathrm{c}}^{2}-1} / \Gamma_{\mathrm{c}}$, we obtain from (B.9) that

$t_{\theta} \leq \frac{t-\frac{D}{\mathrm{c}}+\left(\frac{R_{\mathrm{c}}}{\mathrm{c}}-t_{\mathrm{c}} \beta_{\mathrm{c}}\right) \cos \theta}{1-\beta_{\mathrm{c}} \cos \theta}$.

Applying (49) we get

$t_{\theta} \leq t_{\mathrm{c}}+\frac{R_{\mathrm{c}}}{c} \frac{p+\cos \theta}{1-\beta_{\mathrm{c}} \cos \theta}$

To focus on the effect of variation of the Lorentz factor, we require that all photons observed at the assigned time must be those emitted after the deceleration starts and before the expansion stops, i.e.,

$t_{\theta} \geq t_{\mathrm{c}}$ 
Y.-P. Qin: Emission and absorption lines of gamma-ray bursts, Online Material p 5

and

$\Gamma\left(t_{\theta}\right)>1$.

Combining (C.2) and (C.3) we get

$p \geq 0$.

One can verify by applying (44) and (45) that, when

$k^{7 / 3}>t_{\mathrm{c}}+\frac{R_{\mathrm{c}}}{c} \frac{p+\cos \theta}{1-\beta_{\mathrm{c}} \cos \theta}$,

then (C.4) would be satisfied. Taking $\theta=0$ we get

$k^{7 / 3}>t_{\mathrm{c}}+\frac{R_{\mathrm{c}}}{c} \frac{1+p}{1-\beta_{\mathrm{c}}}$.

Applying (45) we arrive at

$k>\Gamma_{\mathrm{c}}\left[\frac{R_{\mathrm{c}}(1+p)}{c\left(1-\beta_{\mathrm{c}}\right)\left(\Gamma_{\mathrm{c}}^{7 / 3}-1\right)}\right]^{3 / 7}$. 